\title{
Inhibition Underlies Fast Undulatory Locomotion in Caenorhabditis elegans
}

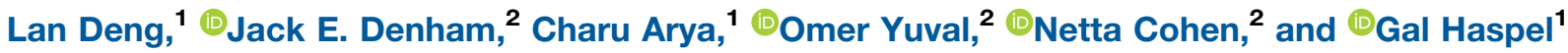

\author{
https://doi.org/10.1523/ENEURO.0241-20.2020
}

${ }^{1}$ Federated Department of Biological Sciences, New Jersey Institute of Technology and Rutgers University, Newark, NJ 07102 and ${ }^{2}$ School of Computing, University of Leeds, Leeds LS2 9JT, United Kingdom

\begin{abstract}
Inhibition plays important roles in modulating the neural activities of sensory and motor systems at different levels from synapses to brain regions. To achieve coordinated movement, motor systems produce alternating contractions of antagonist muscles, whether along the body axis or within and among limbs, which often involves direct or indirect cross-inhibitory pathways. In the nematode Caenorhabditis elegans, a small network involving excitatory cholinergic and inhibitory GABAergic motoneurons generates the dorsoventral alternation of body-wall muscles that supports undulatory locomotion. Inhibition has been suggested to be necessary for backward undulation because mutants that are defective in GABA transmission exhibit a shrinking phenotype in response to a harsh touch to the head, whereas wild-type animals produce a backward escape response. Here, we demonstrate that the shrinking phenotype is exhibited by wild-type as well as mutant animals in response to harsh touch to the head or tail, but only GABA transmission mutants show slow locomotion after stimulation. Impairment of GABA transmission, either genetically or optogenetically, induces lower undulation frequency and lower translocation speed during crawling and swimming in both directions. The activity patterns of GABAergic motoneurons are different during low-frequency and high-frequency undulation. During low-frequency undulation, GABAergic VD and DD motoneurons show correlated activity patterns, while during high-frequency undulation, their activity alternates. The experimental results suggest at least three non-mutually exclusive roles for inhibition that could underlie fast undulatory locomotion in C. elegans, which we tested with computational models: cross-inhibition or disinhibition of body-wall muscles, or neuronal reset.
\end{abstract}

Key words: C. elegans; GABA; inhibition; locomotion; model; motoneuron

\section{Significance Statement}

Inhibition serves multiple roles in the generation, maintenance, and modulation of the locomotive program and supports the alternating activation of antagonistic muscles. To better understand the role of inhibition in locomotion, we used Caenorhabditis elegans as an animal model, and challenged a prevalent hypothesis that cross-inhibition supports the dorsoventral alternation only during backward locomotion. We find that inhibition is not necessary for muscle alternation during slow undulation in either forward or backward locomotion; however, it is crucial to sustain rapid dorsoventral alternation. We combined behavior analysis and calcium imaging of motoneurons and muscle cells with computational models to test hypotheses for the role of inhibition in locomotion.

\section{Introduction}

Alternating activation of antagonistic muscles is ubiquitous in motor programs. During locomotion, limbed animals alternate flexor-extensor muscles and typically their

Received June 8, 2020; accepted December 1, 2020; First published December 15, 2020.

The authors declare no competing financial interests. left and right counterparts, while limbless locomotion involves antagonistic axial muscles that generate body bending. The generation of an alternating pattern often involves direct or indirect inhibitory pathways to the

Author contributions: L.D., N.C., and G.H. designed research; L.D., J.D., C.A., and G.H. performed research; L.D., O.Y., and G.H. analyzed data; L.D., N.C., and G.H. wrote the paper. 
antagonistic pattern generator or motor pool (Kiehn, 2011, 2016). Inhibition serves multiple roles in the generation, maintenance, and modulation of the locomotive program. Hypotheses range from cross-inhibitory induced alternation of antagonists (Sherrington, 1908) to postinhibitory rebound contributing to rhythm generation (Brown, 1912; Friesen, 1994), and concurrence of excitation and inhibition producing neuronal oscillations (Forbes, 1912). Ascending interneurons produce recurrent inhibition of sensory pathways that gate reflex responses and limit firing of central pattern generator (CPG; Wilson, 1961; Marder and Bucher, 2001; Grillner, 2006; Bucher et al., 2015) neurons, while descending interneurons can produce tonic or phasic inhibition that reduces responsiveness and spontaneous locomotion, or stops it all together (Roberts et al., 2008). At the local circuit level, cross-inhibition and reciprocal-inhibition coordinate alternation or, together with postinhibitory rebound, generate or augment network oscillations and a locomotor pattern (Brown, 1912; Satterlie, 1985; Song et al., 2020). Moreover, when excitation is blocked, reciprocal inhibition can sustain a minimal circuit for rhythmic flexorextensor alternation based on postinhibitory rebound (Talpalar et al., 2011). Premotor inhibition is necessary for rapid locomotion in insects (Kohsaka et al., 2014; Gowda et al., 2018), amphibians (Li and Moult, 2012), fish (Liao and Fetcho, 2008; Satou et al., 2009), and mammals (Gosgnach et al., 2006; Zhang et al., 2014; Kiehn, 2016) as well as for rapid respiratory rhythm (Cregg et al., 2017). Across fields disparate from neuroscience, through ecological and genetic networks, to pedestrian and vehicle traffic, negative regulation plays a role in modulating and coordinating the performance of system-parts to overall speed up processes of complex systems (Gershenson and Helbing, 2015). For example, negative autoregulation of gene networks, in which a gene product acts as a repressor of its own transcription, speeds up the response time of gene circuits, while also promoting robustness (Alon, 2007).

In Caenorhabditis elegans, GABAergic motoneurons in the ventral nerve cord have been suggested, based on their morphology, connectivity, and mutant phenotype, to provide cross-inhibition necessary for backward crawling (Mclntire et al., 1993a,b; Schuske et al., 2004). The locomotion circuit is composed of 75 motoneurons, divided by their morphology into 6 excitatory cholinergic and 2

N.C. was supported by the Engineering and Physical Sciences Research Council Grant EP/J004057/1. Some strains were provided by the Cancer Genomics Cloud, which is funded by the National Institutes of Health Office of Research Infrastructure Programs Grant P40 OD010440.

Acknowledgements: We thank Farzan Nadim, Daphne Soares, Andrew Leifer, and Eric Fortune for useful discussions, encouragement, and support; Thomas Ranner for useful and critical discussions; and the National Bioresource Project of Japan and the Takagi and Nakai Laboratories for providing strains.

Correspondence should be addressed to Gal Haspel at haspel@njit.edu.

https://doi.org/10.1523/ENEURO.0241-20.2020

Copyright @ 2021 Deng et al.

This is an open-access article distributed under the terms of the Creative Commons Attribution 4.0 International license, which permits unrestricted use, distribution and reproduction in any medium provided that the original work is properly attributed. inhibitory GABAergic classes that innervate 95 body-wall muscles. The motoneurons synapse onto thin processes called muscle arms that extend from muscle cells onto the ventral cord (White et al., 1976, 1986; Chen et al., 2006; Altun and Hall, 2009; Emmons, 2015). Muscle arms are functionally analogous to vertebrate motoneurons, while $C$. elegans motoneurons are functionally analogous to vertebrate spinal interneurons. GABAergic inhibitory motoneurons (namely, 6 DD and 13 VD motoneurons that innervate dorsal and ventral muscles, respectively) receive inputs only from other motoneurons (White et al., 1976, 1986; Haspel and O'Donovan, 2011; Petrash et al., 2013). Each VD receives input from the dorsal cholinergic motoneurons (DA, DB, and AS) that innervate the opposing muscle cells, while DD motoneurons receive input from the ventral cholinergic motoneurons (VA and VB; White et al., 1976, 1986; Haspel and O'Donovan, 2011), through the cholinergic receptor LGC-46 (Liu et al., 2020). In addition to their synaptic output at neuromuscular junctions, opposing DD and VD motoneurons also innervate each other, whereas VD motoneurons also innervate the local VA and VB (White et al., 1986; Haspel and O'Donovan, 2011) and the locomotion interneurons AVA (Liu et al., 2020).

Mutant strains of $C$. elegans that are defective in any part of their GABAergic transmission (the so-called shrinker mutants) respond to anterior noxious stimuli, such as a harsh touch to the head, with coactivation instead of alternation of antagonistic dorsoventral muscle, producing a shrinking response instead of the rapid reverse undulation seen in the wild type. The mutant animals as well as animals with laser-ablated GABAergic motoneurons, shorten the anterior portion of their body and have been reported not to move backward while performing normal forward crawling, albeit with decreased undulatory amplitude (Mclntire et al., 1993a,b). Hence, it was suggested that GABAergic motoneurons mediate dorsoventral cross-inhibition during backward locomotion (Mclntire et al., 1993b) or direction change (Schuske et al., 2004). According to this hypothesis, when an excitatory cholinergic A motoneuron (e.g., VA) activates postsynaptic muscle arms, it also activates an inhibitory GABAergic motoneuron (e.g., DD) that prevents excitation of the opposing muscle. Shrinking may be caused by the coactivation of dorsal and ventral A-type (DA and VA) motoneurons when initiating backward locomotion or by overlapping activity of locomotion interneurons during a change of direction. An alternative hypothesis suggests that neural, rather than muscular inhibition plays an important role. In a computational model that tested this hypothesis, abolishing neural inhibition from VD to VB is detrimental to rapid, but not to slower, undulation (Boyle et al., 2012).

Here, to assess the relative contributions of these hypotheses, we quantified the response to harsh touch and spontaneous crawling and swimming behaviors of different GABA transmission mutants as well as an optogenetic strain in which GABAergic motoneurons can be inactivated acutely. We recorded the activity of GABAergic motoneurons with calcium imaging in behaving animals during lowfrequency and high-frequency undulation. Finally, we 
suggested competing hypotheses for the roles of inhibition in C. elegans locomotion and tested them with computational models.

\section{Materials and Methods}

\section{C. elegans strains}

We maintained $C$. elegans strains under standard laboratory conditions on nematode growth medium (NGM) agar plates with OP-50-1 Escherichia coli bacterial lawn at $20^{\circ} \mathrm{C}$ (Brenner, 1974; Corsi et al., 2015). All animals used in the experiments were hermaphrodites.

The $C$. elegans strains used in this study were N2 (wild type), CB120, CB1562, CZ1632, TM5487, TM5916, TOL11, TOL12, TOL15, and VC1433. We obtained N2 (a reference laboratory strain considered as wild type), VC1433 [unc-25 (ok1901) III], CB1562 [vab-7(e1562) III], CB120 [unc-4(e120) II], and CZ1632 (juls76 [unc-25p::GFP + lin-15(+)]II) strains from the Caenorhabditis Genetic Center of University of Minnesota (https://cgc.umn.edu/), and TM5916 [unc46 (tm5916)]V and TM5487 [unc-49 (tm5487)]III from the National Bioresource Project of Japan (https://shigen. nig.ac.jp/c.elegans/top.xhtml). We generated TOL11 (aat/s11 [myo-3p::R-CaMP2; unc-25p::G-CaMP6; unc-47p::ChR2:: P2A::DsRed2; odr-1p::DsRed]III and TOL12 (aat/s12[unc47p::Arch::GFP, myo-2p::mCherry]) by integrating the extrachromosomal arrays in jqEx456 [myo-3p::R-CaMP2; unc-25p:: G-CaMP6; unc-47p::ChR2::P2A::DsRed2; odr-1p::DsRed] (Inoue et al., 2015), and OKA074 (ncEx2351 [unc-47p::Arch:: gfp, myo-2p:::mCherry]; Okazaki et al., 2012) into the animals' genome via UV irradiation methods, respectively; then we back-crossed the integrated strains with wild-type N2 for at least five generations and screened for the homozygous (Ahringer, 2006). We generated TOL15 by crossing TOL14 (aat/s4 [myo3p::n/s:::RCaMP2]V) and ZW495 (zw/s132 [myo3p::GCamp2 + lin-15(+)]) and screening for homozygotes for aatls4 and zw/s132.

\section{Free locomotion behavioral tracking}

We recorded the free crawling and swimming of wildtype, GABA transmission knock-outs (VC1433, TM5487, TM5916), and an optogenetic strain (TOL12) on NGM agar plates or in NGM buffer solution using a static multi-worm tracker. The tracker was composed of three major parts. From top to bottom, a camera (Basler ace acA4024-29um) connected to a fixed focal length lens (C Series 5MP $35 \mathrm{~mm}$ 2/3" fixed focal length lens, Edmund Optics) with an infrared cutoff filter (M25.5 $\times 0.5$ Mounted IR cutoff Filter, Edmund Optics), a specimen stage for plates or slides, and an infrared LED light (M850L3, Thorlabs) mounted with a collimation adaptor (COP1-B-Collimation Adaptor, Thorlabs).

One day before recording, we transferred fourth larval stage (L4) animals onto new NGM plates with OP-50-1 E. coli bacterial lawn so that experimental animals would be first-day young adults. During tracking, at least 30 animals were freely moving on $35 \mathrm{~mm}$ NGM plates without bacterial lawn or in NGM solution between a microscope slide and a coverslip, $1 \mathrm{~mm}$ apart. We recorded at least nine videos with Pylon Viewer (Basler pylon Camera Software Suite) at 25 frames per second (fps) for $15-20 \mathrm{~s}$. During video acquisition, we cropped the video frame dimensions to $\sim 2000 \times 2000$ pixels.

One day before behavioral tracking of the optogenetic strain TOL12 [expressing Archaerhodopsin-3 (Arch3; Chow et al., 2010) in GABAergic neurons], we transferred L4 animals onto NGM plates that had been freshly seeded with $50 \mathrm{mM}$ all-trans-retinal (ATR) in OP-50-1 E. coli. To allow the animals to incorporate ATR, we kept the plates in the dark at $20^{\circ} \mathrm{C}$ for $24 \mathrm{~h}$. Animals fed with ATR but recorded in the dark (under infrared LED) were tracked as a negative control group, and the same animals under limecolor LED light that activated Arch3 were the experimental group. As another negative control group, TOL12 animals that were not fed with ATR were not affected by the limecolor activation light of the optogenetic protein during crawling or swimming ( $n=30$ animals from three plates). We used lime-color light (M565L3, $565 \mathrm{~nm}$ LED with a Collimator), which is the optimal activation light of Arch3 (Mattis et al., 2011; Okazaki et al., 2012), with a working illumination power of $10 \mathrm{~mm} / \mathrm{cm}^{2}$. To prevent desensitization of TOL12 to the activation light, the maximum time of light exposure was $1 \mathrm{~m}$ (Okazaki et al., 2012). The rest of the operations and software settings were the same as the behavior tracking aforementioned.

\section{Head and tail harsh touch experiments}

We transferred L4 animals to new OP-50-1 E. coliseeded NGM plates $1 \mathrm{~d}$ before the experiment so that all assays were done on young adult hermaphrodite animals. During the experiment, we transferred animals onto $60 \mathrm{~mm}$ NGM plates without bacterial lawns. We touched the head or tail of the animals using a blunt glass probe while they were crawling forward. We acquired the videos continuously with a CMOS camera (U3CMOS14000KPA, ToupTek) and acquisition software (ToupView, http://www.touptek.com) at $30 \mathrm{fps}$ and $1048 \times 822$ pixels greyscale during several stimulations of different animals. We further trimmed these videos into clips with one stimulation each by video editing software Premiere Pro (Adobe) or Shotcut (https://shotcut. org/). We also tested the response of wild-type young adult hermaphrodites to a weak chemical repellant: $0.1 \%(\mathrm{v} / \mathrm{v})$ sodium dodecyl phosphate (SDS) off-food, and a strong chemical repellant: $50 \mathrm{mM}$ copper sulfate $\left(\mathrm{CuSO}_{4}\right)$ on a thin layer of food (Hilliard et al., 2004; Hart, 2006; Ezcurra et al., 2011). To make sure that harsh touch does not mechanically cause changes in body length we paralyzed young adult animals with $1 \mathrm{mM}$ tetramisole hydrochloride (Sigma-Aldrich, $10 \mathrm{~m}$ immersion). We touched the head or tail of paralyzed animals using a blunt glass probe, acquired videos, and analyzed body length as described above. There was no significant change in body length of paralyzed animals (99 $\pm 1 \%$; $p=0.21$ paired $t$ test; Extended Data Fig. 2-1).

\section{Behavioral tracking analysis}

We processed tracking videos of crawling and swimming using Tierpsy Tracker v1.4.0, a multi-worm behavior tracker software (https://github.com/ver228/ 
tierpsy-tracker/releases/; Javer et al., 2018). Then, we analyzed hdf5 file generated by Tierpsy with custom programs (Extended Data 1) written in MATLAB (MathWorks) to extract behavior parameters and to plot curvature kymograms (the code for kymograms includes the function smoothn by Garcia, 2010).

Each video had between 10 and 30 animals each assigned with an index by Tierpsy. We analyzed the translocation speed and undulation frequency during forward and backward locomotion from the midbody of the animals as well as primary wavelength and maximal amplitude. Tierpsy computes the undulation frequency using the waveform frequency from the largest peak via the Fourier transform over a time window.

We used Tierpsy Tracker v1.4.0 to extract the aforementioned kinetic parameters from trimmed video clips before and after head or tail stimulation. We calculated the means of these four parameters within $5 \mathrm{~s}$ before and $5 \mathrm{~s}$ after the stimulation from trimmed videos of head and tail stimulation experiments.

\section{Changes in body length}

We analyzed the body length for each stimulation direction of N2 strain and of each of the GABA transmission knock-out strains, as well as head stimulation of vab-7 mutant animals (CB1562) and tail stimulation of unc-4 mutant animals (CB120). We measured the body length at different times in the trimmed video clips: before the harsh stimulation, immediately after the stimulation, as well as $0.5,1$, and $2 \mathrm{~s}$ after the stimulation. We used 'Freehand Line' tool in ImageJ (https://imagej.nih.gov/ij/) to measure the body length of animals. We expressed the body length as the percentage of prestimulus length and plotted the means of normalized body lengths for each strain at each time point.

\section{Calcium imaging in a microfluidic device}

To repeatedly measure activity at particular phases of the locomotor cycle, we placed young adult animals in a waveform silicon microfluidic device with channels designed to restrict their motion to a pattern that mimics undulatory locomotion. The cycle was fixed in space and predictable at any location along the path. The wavelength, amplitude, and width of the channel were 686.5, 322.5, and $133.5 \mu \mathrm{m}$, respectively. To unify the frame of reference, we arbitrarily designated the phase of a maximal dorsal bend as $90^{\circ}$ and a maximal ventral bend as $270^{\circ}$ and defined the rest of the phase positions accordingly. We presented collated recordings during forward locomotion from motoneurons and body-wall muscle with phase (rather than time) being the common frame of reference.

We transferred healthy fourth larval stage (L4) animals to new plates seeded with OP-50-1 E. coli $1 \mathrm{~d}$ before imaging. We added drops of methylcellulose solution onto the face of a microfluidic device, and then transferred the animals into these drops. To seal the microfluidic device, we flipped covered it with an $18 \mathrm{~mm}$ diameter round coverslip, flipped the coverslip so the device is on top for the inverted microscope and sealed by pressing on the device. The transgenic animals could crawl freely inside the channels, and we manipulated their undulation frequency by changing the ambient viscosity. We used $0.5 \%$ and $3 \%(\mathrm{w} / \mathrm{w})$ methylcellulose in NGM buffer while recording calcium level changes in GABAergic motoneurons (TOL11), or from green fluorescent protein (GFP) as a negative control calcium-insensitive fluorescent protein to determine motion artifact (CZ1632), and 1.5\% (w/w) methylcellulose to record calcium level changes in bodywall muscles (TOL15). We only recorded calcium level changes in animals that moved smoothly in the microfluidic channel.

To image and record the fluorescence change of GCaMP or GFP, we used an inverted microscope (Olympus IX73) with a 40×/NA0.95 UPlanSApo objective and an sCMOS camera (Hamamatsu Orca Flash 4.0) and a solid-state excitation light source (X-Cite 120LED) with a dual-band filter set (Chroma 59022 ET-EGFP/mCherry), and a dual camera image splitter (TwinCam, Cairn Research with a T565lpxr dichroic beam splitter). We used MicroManager (v1.4; https://micro-manager.org/) to acquire multiple videos of animals moving through different parts of the channel (i.e., different locomotor phases). We saved videos as separate tiff files $(2048 \times 2048$ pixel resolution) at a frame rate of $100 \mathrm{fps}$ with 10-ms exposure for animals crawling in $0.5 \%$ (w/w) methylcellulose, and $50 \mathrm{fps}$ with 20-ms exposure for animals crawling in 1.5\% (w/w) and 3\% (w/w) methylcellulose. We did not use NGM with $<0.5 \%$ (w/w) methylcellulose because it resulted in discontinuous and less smooth locomotion. Between acquisitions, we moved the microscope stage manually to follow animals when they moved out of view. We recorded activity in muscle cells and motoneurons in the mid-body: dorsal muscle cells 9-21 $(n=24)$, ventral muscle cells 9-21 ( $n=27)$, DD motoneurons $2-5(n=26)$, and VD motoneurons 3-10 $(n=59)$.

We used a customized MATLAB program (Extended Data 1) to analyze the change in fluorescence intensity of identifiable body-wall muscle cells or somata of GABAergic motoneurons. For each frame, we measured the mean fluorescence intensity of the top $50 \%$ pixels within the manually traced region of interest (ROI) around the cell of interest $\left(F_{\text {top50\% }}\right)$. We then calculated and subtracted the median intensity of the frame as the background $\left(\mathrm{F}_{\mathrm{BG}}\right)$. Finally, we demeaned the value by dividing it by the mean fluorescence signal of this cell in the video of $n$ frames: $\Delta F / F=\left(F_{\text {top50\% }}-F_{B G}\right) /\left(\Sigma\left(F_{\text {top50\% }}-F_{B G}\right) / n\right)$. Our MATLAB code provides a graphical user interface (GUI) to track the cell as it moves across the field of view, following a trajectory that is set by the waveform channel, to mark the location of $90^{\circ}$ (maximal dorsal bend) or $270^{\circ}$ (maximal ventral bend), and to trace $\Delta \mathrm{F} / \mathrm{F}$ against time or undulatory phase. We categorized traces from GABAergic motoneurons into low-frequency and high-frequency undulation of crawling inside the microfluidic channel (with $0.6 \mathrm{~Hz}$ as cutoff, see Results), the undulation frequency was calculated as the fraction of locomotor cycle traveled by the cell of interest divided by the elapsed time. We collated and plotted all traces of $\Delta F / F$ against the phase of each type of cell at $5^{\circ}$ bins. We corrected the soma position of motoneurons to their neuromuscular postsynaptic 
position according to the published perimotor locations (Chen et al., 2006; Haspel and O'Donovan, 2011). We removed videos or parts of videos in which the cell of interest went out of focus or if we could not positively identify it.

\section{Computational modeling}

To probe different hypotheses about neural control of locomotion and test their consequences in different fluid environments, we used an integrated neuromechanical model. Our model nematode consists of a smooth, continuous mechanical body, driven by neuromuscular activation, and subject to fluid drag forces (Denham et al., 2018). Our models aim to address the phenotypes of GABA transmission mutants during forward or backward locomotion, rather than directional changes or the shrinker phenotype. Specifically, we focus on our results showing suppressed undulation frequencies in GABA mutants and suggesting that this phenotype is caused by defects in the ventral nerve cord circuitry, rather than by defects in premotor interneurons. To this end, we adapted an existing model of forward locomotion control (Denham et al., 2018). In particular, we explored two different paradigms of neural control separately to parse their qualitative differences: a proprioceptively driven model and a feedforward model, mimicking CPG control (Denham et al., 2018). We subjected each model to different perturbations and explored the behavior generated in our neuromechanical framework in virtual liquid and agar-like conditions. Model code and parameters are given in Extended Data 1 and Extended Data Figure 8-1.

\section{Neuromuscular model framework}

Whether under CPG-driven, or proprioceptively-driven, paradigms of locomotion control, our models of neural control generate a neural activation function that acts continuously along the body. We denote the neural activation by $A(u, t)$, where $u \in[0,1]$ represents the distance along the midline of the nematode from head $(u=0)$ to tail $(u=1)$, and $t$ is time. This neural activation function corresponds to the difference between dorsal and ventral muscle inputs that arise from cholinergic B-type excitatory and GABAergic D-type inhibitory motoneurons on either side of the body. The mechanical component of the model is a flexible viscoelastic shell that is actuated by a spatially continuous muscle activation function, $\beta(u, t)$, which is, in turn, derived from the neural activation function, $A(u, t)$.

Our approach to modeling is parsimonious, with the aim of maximizing the explanatory power of the model with respect to the specific model hypotheses, and the effect of inhibition on undulation frequency. Therefore, asymmetries in dorsal versus ventral activation (PierceShimomura, 2008), detailed muscle anatomy (e.g., around the pharynx and vulva), more detailed representations of neural connectivity (Haspel and O'Donovan, 2011), cuticle viscosity, muscle tone modulations, and rigorous models of the external fluid dynamics are all neglected in this study. We also note that model results are qualitatively similar when using either a fixed or decaying amplitude of contractions along the body (see Boyle et al., 2012;
Cohen and Ranner, 2017; Denham et al., 2018). Accordingly, under both control paradigms, we consider uniform neural and muscle properties along the entire body.

\section{Paradigm 1: CPG control}

Following Denham et al. (2018) and in the absence of detailed CPG mechanisms, we model the essential feature of feedforward control, namely, the existence of endogenous, central pattern generation that produces neural oscillations at a characteristic frequency and is embedded in a biomechanical body. We therefore model the neural activation by a retrograde traveling sine wave of unit amplitude with period, $T_{\mathrm{f}}$, and wavelength, $\lambda_{\mathrm{f}}$, that activates the muscles continuously along the body. Thus, the neural activation function takes the following form:

$$
A(u, t)=\sin \left(2 \pi u L / \lambda_{\mathrm{f}}-2 \pi t / T_{\mathrm{f}}\right),
$$

where $L$ denotes the length of the body. While abstract, our CPG model is well suited to address the role of muscle inhibition, which has been and remains the main focus of research on the role of GABAergic motoneurons in $C$. elegans locomotion (Mclntire et al., 1993b; Schuske et al., 2004; Wu et al., 2018). As such, however, this model is agnostic about the role of neural inhibition in pattern generation itself.

\section{Paradigm 2: proprioceptive control}

In C. elegans, proprioceptive control of forward locomotion is mediated by B-type excitatory motoneurons (Wen et al., 2012; Fouad et al., 2018). Based on the neural circuitry, anatomy, and ablation results (Chalfie et al., 1985; White et al., 1986; Chen et al., 2006) and consistent with previous models of forward locomotion (Niebur and Erdös, 1991; Bryden and Cohen, 2008; Cohen and Boyle, 2010; Boyle et al., 2012) in our model, B-type motoneurons receive a descending locomotion command input and local inhibitory input; internally, they are driven by a spatially extended proprioceptive input (Denham et al., 2018). For an in depth discussion of this class of models, see relevant reviews (Cohen and Boyle, 2010; Cohen and Sanders, 2014; Cohen and Denham, 2019).

\section{Model B-type motoneurons}

Following Boyle et al. (2012) and informed by electrophysiological recordings of bistable RMD head motoneurons (Mellem et al., 2008), we model excitatory ventral B-type (VB) and dorsal B-type (DB) motoneurons as bistable switches with binary states, $\{0,1\}$. Each neuron state is defined at every point along the body and denoted by $V_{\mathrm{VB}}(u, t)$ and $V_{\mathrm{DB}}(u, t)$. Neuronal bistability is implemented as distinct ON and OFF thresholds on the ventral $(\mathrm{V})$ and dorsal (D) sides, $\theta_{k}^{\mathrm{ON}}$ and $\theta_{k}^{\mathrm{OFF}}(k \in\{\mathrm{V}, \mathrm{D}\})$, which need to be crossed for a neuronal state change of VB or DB to occur (akin to Boyle et al., 2012). Both VB and DB receive the same constant descending input from the AVB premotor interneurons, $I_{A V B}$, which may be absorbed into the thresholds for parsimony. Proprioceptive inputs provide a feedback loop that couples the neural control and biomechanics. A proprioceptive input, $I_{\kappa}(u, t)$, is defined for the dorsal side such that the input on the ventral side at the 
same position and time is $-I_{\kappa}(u, t)$. Combined in our biomechanical framework, neuronal bistability and spatially extended proprioceptive inputs are sufficient to qualitatively capture observed gait modulation in response to changes in the external fluid environment, typically measured in terms of the frequency and waveform of undulation (Berri et al., 2009; Fang-Yen et al., 2010).

\section{Model inhibition}

As the limiting timescales of the dynamics are mechanical, we assume that D-type inhibitory motoneurons, with states $V_{\mathrm{VD}}(u, t)$ and $V_{\mathrm{DD}}(u, t)$, respond instantaneously to excitation from B-type motoneurons on the opposite side of the body. Thus, if DB (or VB) is on at some point along the body, VD (or DD) is activated. Following Boyle et al. (2012) and the published connectivity (Chen et al., 2006), we include VD-to-VB inhibition: thus, VB is inhibited by VD (when $D B$ is active), producing an input current, $l_{\mathrm{VD}}(u, t)=w V_{\mathrm{VD}}(u, t)$. In our implementation of the wildtype model, we set the inhibition strength, $w$, such that the thresholds of VB and DB collapse to a single bistable switch when DB (hence also VD) is active. The corresponding reduced model, with only a single bistable switch, is presented and analyzed in depth in Denham et al. (2018).

Combining the above model components, VB and DB state switches are given by the following relations:

$$
\begin{array}{lll}
V_{\mathrm{DB}}(u, t) \rightarrow 1 \quad \text { if } & I_{\mathrm{AVB}}-I_{\kappa}>\theta_{\mathrm{D}}^{\mathrm{ON}}, \\
V_{\mathrm{DB}}(u, t) \rightarrow 0 & \text { if } & I_{\mathrm{AVB}}-I_{\kappa}<\theta_{\mathrm{D}}^{\mathrm{OFF}}, \\
V_{\mathrm{VB}}(u, t) \rightarrow 1 & \text { if } & I_{\mathrm{AVB}}+I_{\kappa}-I_{\mathrm{VD}}>\theta_{\mathrm{V}}^{\mathrm{ON}}, \\
V_{\mathrm{VB}}(u, t) \rightarrow 0 & \text { if } & I_{\mathrm{AVB}}+I_{\kappa}-I_{\mathrm{VD}}<\theta_{\mathrm{V}}^{\text {OFF }} .
\end{array}
$$

The state of a B-type neuron is driven by changes in proprioceptive input current, $I_{\kappa}(u, t)$, which we define here as the mean of the signed body curvature, $\kappa(u, t)$, over a specified proprioceptive range, $\Delta(u)$, represented as a fraction of the body length:

$$
I_{\kappa}(u)=\frac{\int_{u}^{u+\Delta(u)} \kappa\left(u^{\prime}\right)\left|\boldsymbol{x}_{u}\left(u^{\prime}\right)\right| d u^{\prime}}{\int_{u}^{u+\Delta(u)}\left|\boldsymbol{x}_{u}\left(u^{\prime}\right)\right| d u^{\prime}},
$$

where $\boldsymbol{x}$ denotes the coordinates of a point along the body in the lab frame, the subscript, $u$, denotes a partial derivative, and time dependence is implicit. This encoding of body posture is fed back to the motoneurons, thus closing the brain-body-environment loop. The above input current represents the effective stretch of the dorsal side of the body at that time. Here, we keep $\Delta(u)>0$ which by our convention corresponds to a strictly posterior proprioceptive range, though $\Delta(u)<0$, and combinations of anterior and posterior range are also possible (see Denham et al., 2018). For $u$ sufficiently close to the tail, the proprioceptive range terminates at the tail, therefore decreasing linearly with $u$. Hence the denominator in Equation 3 normalizes by this range, effectively making posterior neurons more sensitive to stretch "density" to compensate for the reduced range. In all simulations, we set the proprioceptive range to half the body length, posteriorly to the muscle coordinate (Boyle et al., 2012; Denham et al., 2018).

As our biomechanical model uses a midline parametrization to determine the internal forces driving body curvature, our neural activation function sums the contributions of local neurons from both sides of the body, weighted by excitatory and inhibitory neuromuscular junction weights, $w_{\mathrm{NMJ}}^{\mathrm{exc}}, w_{\mathrm{NMJ}}^{\text {inh }}$, according to

$$
\begin{aligned}
A(u, t)= & w_{\mathrm{NMJ}}^{\mathrm{exc}}\left(V_{\mathrm{DB}}(u, t)-V_{\mathrm{VB}}(u, t)\right)- \\
& w_{\mathrm{NMJ}}^{\text {inh }}\left(V_{\mathrm{DD}}(u, t)-V_{\mathrm{VD}}(u, t)\right),
\end{aligned}
$$

to define our activation, $A(u, t)$, which then drives the muscle via Equation 5 below.

\section{Model muscles}

Under proprioceptive control, body-wall muscles are modeled by a leaky integration equation that converts the neural activation function at every point along the body, $A(u, t)$, to a mechanical torque. Specifically, muscles receive local inputs from ventral and dorsal motoneurons and output the muscle activation, $\beta(u, t)$, acting on the animal's midline. The muscle model contains two free parameters: a muscle time scale, $\tau_{\mathrm{m}}$, set to $100 \mathrm{~ms}$ (Extended Data Fig. 8-1) in the wild type, and an amplitude or maximum curvature $\left(10 \mathrm{~mm}^{-1}\right.$ in the wild type; for discussion, see Denham et al., 2018). Thus, given the neural activation, $A(u, t)$, the muscle response is given by

$$
\tau_{\mathrm{m}} \frac{\mathrm{d} \beta(u, t)}{\mathrm{d} t}=-\beta(u, t)+\beta_{0} A(u, t),
$$

where the muscle activation, $\beta(u, t)$, corresponds to a preferred curvature (akin to a rest length of a spring) and $\beta_{0}$ sets the curvature amplitude (Eq. 8 below).

In the case of CPG control, and to accommodate the disinhibition of body-wall muscles, we permit (under one hypothesis) the muscle activation in the wild-type to depolarize more sharply than in GABA mutants. We therefore set the muscle activation to

$$
\beta(u, t)=\frac{\beta_{0} \tanh (\alpha A(u, t))}{\tanh (\alpha)},
$$

with the steepness, $\alpha$, corresponding to the strength of the muscle activation following disinhibition and where the denominator normalizes the amplitude of the muscle activation.

\section{Biomechanics}

Our model of the $C$. elegans body consists of a thin incompressible viscoelastic shell, subject to internal pressure and external forces (see Cohen and Ranner (2017) and Ranner (2020) for a detailed description of the body 
mechanics, mathematical derivation and numerical methods). At each point along the body, we assume the radius of the nematode's body is fixed in time, which allows us to collapse all internal and external forces onto the midline (Denham et al., 2018; Ranner, 2020). Four free parameters modulate the biomechanical properties of the body and its interaction with the environment. The body is parametrized by its effective Young's modulus, $E$ (the effective elastic resistance to bending or body stiffness, set to $100 \mathrm{kPa}$ in the wild type), and an internal viscosity (the body damping in response to bending), which we neglect for simplicity. Environmental forces are modeled by resistive force theory and parametrized by tangential and normal drag coefficients, $K_{\tau}$ and $K_{v}$, that act against the cuticle to resist the motion in the respective directions, denoted by unit vectors, $\tau$ and $v$ (Berri et al., 2009; Boyle et al., 2012; Denham et al., 2018). Accordingly, the resistive forces are given by

$$
\boldsymbol{F}_{\mathrm{env}}=K_{\tau} v_{\tau} \tau+K_{v} v_{v} v,
$$

where $v_{\tau}$ and $v_{v}$ represent the tangential and normal components of the velocity of a point on the surface of the body. This allows us to model both Newtonian and linear viscoelastic environments in the low Reynolds number regime. Our simulations here mimic two environmental conditions: swimming in liquid (buffer solution) and crawling on agar. The linear viscoelastic approximation of the fluid dynamics was validated for these conditions (Berri et al., 2009), provides computational efficiency, and is sufficient to reproduce the experimentally observed gait transition under models of feedback control (Boyle et al., 2012; Denham et al., 2018).

The model equations balance internal and external forces and torques subject to mass conservation within the nematode's body and an inextensibility closing constraint (Cohen and Ranner, 2017; Ranner, 2020). To produce a body bend, the signed midline curvature, $\kappa(u, t)$, is driven by the muscle activation, $\beta(u, t)$ (here, a torque that is represented as a preferred body curvature), which in turn is driven by neural activation, $A(u, t)$, in either the CPG or proprioceptive control paradigm. By convention, positive and negative $\beta(u, t)$ and $\kappa(u, t)$ correspond to dorsal and ventral excitation and body curvature, respectively. The timescale of undulation depends on both the elasticity of the body, $E$, and the resistivity of the environment (Cohen and Ranner, 2017; Denham et al., 2018). The balance of forces is summarized as follows:

$$
\boldsymbol{F}_{\mathrm{env}}-\frac{1}{\left|\boldsymbol{x}_{u}\right|}(p \tau)_{u}+\frac{1}{\left|\boldsymbol{x}_{u}\right|}\left(\frac{1}{\left|\boldsymbol{x}_{u}\right|} E I_{2}(\kappa-\beta)_{u} \boldsymbol{v}\right)_{u}=0,
$$

where $p$ represents the nematode's internal pressure which acts as a line tension along the midline of the nematode and $I_{2}$ denotes the second moment of area. Zero force and zero torque are enforced at the boundaries, such that $\beta(u, t)=\kappa(u, t)$ at both the head and tail ends of the body $(u=0,1)$. We note that our biomechanical model is symmetrical across the dorsal and ventral sides.

\section{Model perturbations}

Based on our experimental results, we explore three hypotheses to explain the role of inhibition in sustaining high-frequency locomotion; each of these hypotheses is tested separately in our computational models under purely feedforward (CPG) and purely feedback (proprioceptive) neural control. To qualitatively describe different perturbations, relatively small perturbations are the most useful in that they are restricted to the domain in which the model was set (best matching crawling and swimming behaviors of the wild type). To determine the range of perturbations and their effects, parameter sweeps were performed for hypotheses 1 and 2 (Extended Data Fig. 8-2). Unless otherwise stated, we only knock out one form of inhibition in the model in a given simulation.

Hypothesis 1. Cross-inhibition of the opposing bodywall muscles increases the dorsoventral difference in muscle activation. To implement this hypothesis in our model, we eliminated all muscle inhibition by D-type neurons, leaving neural inhibition intact. In our model, the muscle forcing on both sides of the body is combined into a single muscle activation function acting along the midline of the body. Therefore, the elimination of muscle cross-inhibition by D-type neurons effectively results in reduced activation on the bending side. In practice, we implement this change with a multiplicative constant applied to our preferred curvature:

$$
\beta(u, t) \rightarrow c \beta(u, t),
$$

with $c=1$ in the wild type and $c=0.8$ in the default perturbation. In the absence of muscle cross-inhibition, setting $c<1$ indicates that the muscle on the stretched side remains partially activated (Goodman et al., 2011; Boyle et al., 2012). In our proprioceptively driven model, we set the neuromuscular junctions of B-type and D-type motoneurons such that eliminating muscle inhibition gives an identical outcome (see Extended Data Fig. 8-1).

Hypothesis 2. Disinhibition of body-wall muscles should increase muscle force, immediately after muscle inhibition is released, resulting in a sharper upstroke in muscle activation on the contracting (bending) side of the body. We distinguish between CPG and proprioceptively driven control as follows:

\section{CPG control}

In the wild-type model, we enforce a sharpness in the upstroke of muscle drive using a sigmoidal muscle activation function, which we neglect in the default disinhibition deficient models:

$$
\beta^{\text {hyp2 }}(u, t)=A(u, t)=\sin \left(2 \pi u L / \lambda_{\mathrm{f}}-2 \pi t / T_{\mathrm{f}}\right) .
$$

More generally, for the purpose of parameter sweeps (Extended Data Fig. 8-2), we vary the steepness of the upstroke, $\alpha$, in Equation 6.

\section{Proprioceptive control}

While this could be modeled by a more complex nonlinear muscle model, we reasoned that the effect of a muscle disinhibition can be qualitatively modeled using our linear muscle equation by allowing disinhibition deficient 
models to have a slower muscle timescale on the contracting side of the body. Because of the alternation of muscle contraction, the combined dorsoventral muscle activation, $\beta^{\text {hyp2 }}(u, t)$, of the midline will experience an overall slower effective muscle timescale, $\tau_{\mathrm{m}}^{\text {hyp2 }}$, yielding

$$
\tau_{\mathrm{m}}^{\text {hyp2 }} \frac{\mathrm{d} \beta(u, t)}{\mathrm{d} t}=-\beta(u, t)+\beta_{0} A(u, t),
$$

where $\tau_{\mathrm{m}}^{\text {hyp2 }}=1.2 \tau_{\mathrm{m}}$.

Hypothesis 3. Inhibitory reset of VA and VB by VD is required for rapid swimming. Boyle et al. (2012) hypothesized that nematodes lacking neural inhibition will fail to undulate in water-like environments, in which wild-type nematodes undulate at higher frequency. As this hypothesis depends explicitly on the GABAergic neural circuit (and as our simplistic feedforward model does not take into account individual motoneurons), we only explore this hypothesis in our proprioceptively driven model. In this model, VB activation depends on the state of $\mathrm{DB}$ because of the $\mathrm{DB} \rightarrow \mathrm{VD} \rightarrow \mathrm{VB}$ connectivity. Elimination of neural inhibition implies that VB neurons are not inhibited when DB neurons are active, but that muscle cross-inhibition remains intact. To model defects corresponding to this hypothesis, we set $I_{V D}=0$ at all times. This effectively results in a constant positive offset applied to both thresholds of the VB bistable switch.

\section{Parameter choices for perturbations}

As the change of waveform in GABA mutants is subtle, and extensive literature points only to subtle changes in forward locomotion (Mclntire et al., 1993b; Schuske et al., 2004; Bamber et al., 2005; and our own data herein), we assume that the effect of excitation is much stronger than that of inhibition. Muscle contraction typically leads to, at most, a $20 \%$ change in muscle length (Petzold et al., 2011; Boyle et al., 2012). Therefore, we set $20 \%$ as an upper cap on perturbation strength in all model perturbations. We note that neural activation in our model maps linearly to muscle input and muscle length. Applying the same magnitude perturbations in all cases aids intuition and points to the robustness of the mechanisms investigated in the model. Thus, our choice of $20 \%$ is given as a visual example in the figures for a particular parameter value and does not constitute a quantitative model prediction. Values up to $20 \%$ gave qualitatively similar results (Extended Data Fig. 8-2).

\section{Sample size, experimental design, and statistical analysis}

For free crawling behavior tracking of N2 (wild type), the number of videos, animals, and data points were 12, 235, and 67,209 , respectively, for VC1433, the numbers were 11, 213, and 80,407; for TM5916 the numbers were 11, 255 , and 94,446, and for TM5487 the numbers were 12, 289 , and 121,374 . For free-swimming behavior tracking of N2 (wild type), the numbers of videos, animals, and data points were 46, 707, and 164,057, respectively, for VC1433, the numbers were 39,778 , and 247,656; for TM5916 the numbers were 38,1014 , and 316,094; and for TM5487, the numbers were 34,668 , and 293,482 . For free-crawling behavior tracking of TOL12 without ATR feeding, the number of videos, animals, and data points were 20,375, and 104,098, respectively. For TOL12 that were fed with ATR and tracked in the dark, the numbers were 27, 335, and 98,017; and for TOL12 that were fed with ATR and were exposed to lime-color light during tracking, the numbers were 26,235 , and 66,803 . For freeswimming behavior tracking of TOL12 without feeding ATR, the number of videos, animals, and data points were 20,326 , and 81,291 , respectively. For TOL12 that were fed with ATR in advance and tracked in the dark the numbers were 22, 361, and 97,630; and for TOL12 that were fed with ATR and were exposed to lime-color light during tracking, the numbers were 18,216 , and 48,323 . We collated the means of these parameters from individual animals from all the videos, and then used one-way ANOVA and Tukey's multiple comparison to compare the estimated population means among strains or different conditions.

For the head and tail harsh touch experiments, we obtained nine trimmed videos for each stimulation direction for N2 and GABA transmission knock-out strains (VC1433, TM5916, TM5487) as well as nine trimmed videos for vab-7 mutants (CB1562) of head harsh touch and unc-4 mutants (CB120) of tail harsh touch. We used a paired two-tail $t$ test to determine statistical differences in the parameters before and after stimulation of the same strain, and one-way ANOVA and Tukey's multiple comparison for the parameters of after harsh touch to the head or tail between wild-type and mutant strains, including GABA transmission knock-outs, and vab-7 and unc-4 mutants.

To calculate the linear regressions between frequency and speed during sinusoidal crawling and swimming behaviors, we only used data points for which the primary or secondary wavelengths were extracted by the analysis software (Tierpsy). These data points in crawling behavior comprised $4-23 \%$ of the raw data. The sample sizes of wild type, unc-25, unc-46, and unc-49 during forward crawling were 11935 (23\%), 7760 (14\%), 7773 (12\%), and $5378(7 \%)$, respectively; those during backward crawling were 1260 (11\%), $2319(11 \%), 2552(10 \%)$, and 1714 (4\%), respectively. The data points in swimming behavior comprised $6-54 \%$ of the raw data. The sample sizes of wild type, unc-25, unc-46, and unc-49 during forward swimming were 74,948 (54\%), 46,355 (26\%), 69,682 $(31 \%)$, and $17,015(11 \%)$, respectively; those during backward swimming were 2137 (14\%), 6249 (11\%), $16,728(21 \%)$, and $7297(6 \%)$, respectively. Then, we picked the data points where the path of an undulation cycle (translocation speed $\times$ undulation cycle period) was larger than either the primary or secondary wavelength. Based on this selection criterion, we used $27-54 \%$ of the data points for crawling behavior and $0.4-26 \%$ data points for swimming behavior. The sample sizes of wild type, unc-25, unc-46, and unc-49 during forward crawling comprised 5671 (48\%), 2058 (27\%), 2259 (30\%), and $2313(43 \%)$, respectively; those during backward crawling were $686(54 \%), 1096(47 \%), 1199(47 \%)$, and $856(50 \%)$, respectively. The sample sizes of wild type, unc-25, unc- 

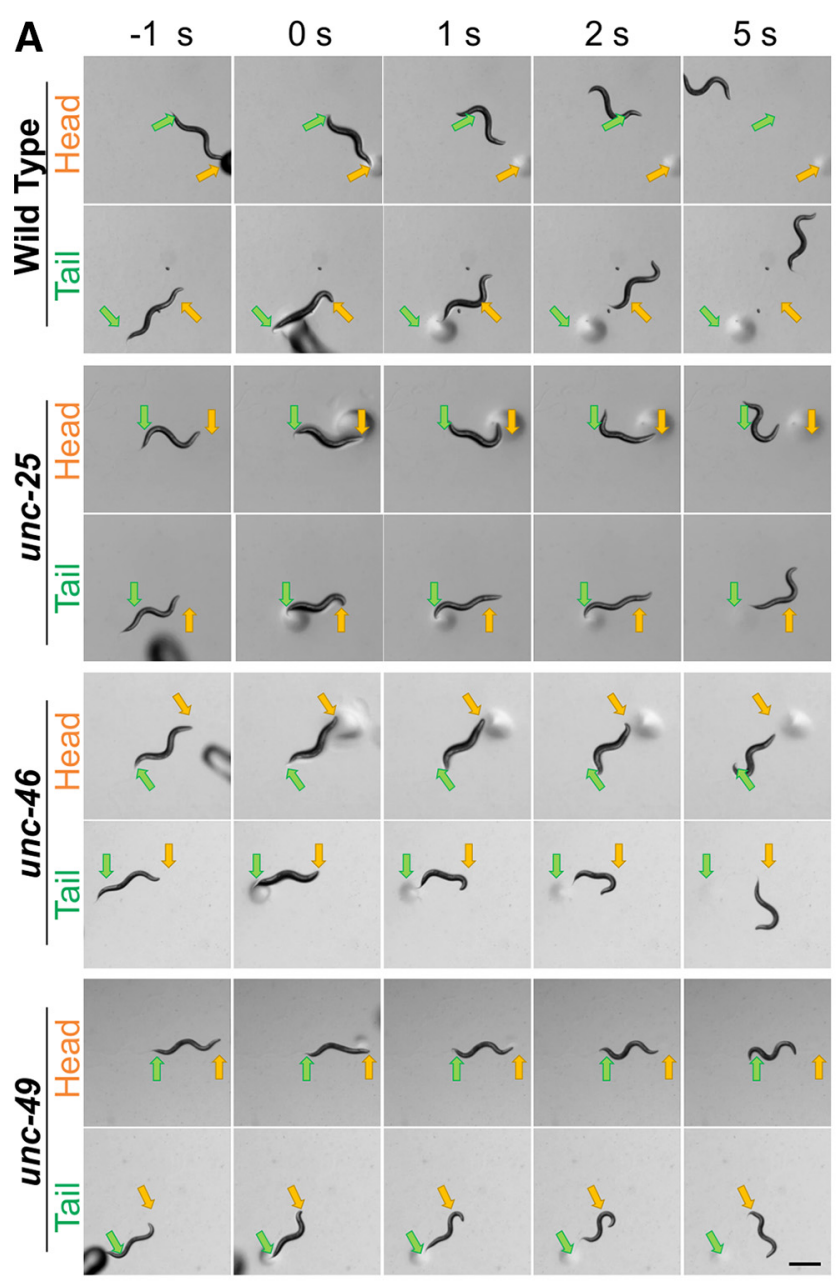

B1

B2
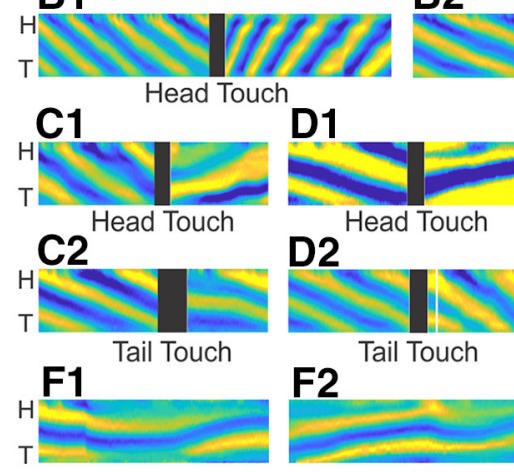

Spontaneous Shrinking Events

Figure 1. Wild-type and GABA transmission knock-outs shrank after harsh touch to the head or tail, while wild-type animals escaped rapidly. $\boldsymbol{A}$, While moving forward, animals were touched by a blunt glass probe as a harsh stimulation to the head (yellow arrow) or tail (green arrow). Wild-type animals briefly shrank immediately after stimulation (compare $0 \mathrm{~s}$ to before stimulation $(-1 \mathrm{~s})$ and rapidly moved away from the stimulus, while GABA transmission knock-outs shrank for a longer time and moved away slowly. Note that by $5 \mathrm{~s}$, all animals had moved away from the stimuli. Common scale bar is $0.5 \mathrm{~mm}$. Dark and circular shadows are the glass probe and the marks it left on agar. After harsh stimulation, wild-type animals $(\boldsymbol{B})$ increased undulation frequency to escape backward (B.1) or forward (B.2). In contrast, all three GABA transmission mutants, unc-25 (C), unc-46 continued

$(\boldsymbol{D})$, and unc-49 $(\boldsymbol{E})$, decreased undulation frequency when they moved away from the stimuli. Moreover, there was a period in which the body posture of these mutants did not change after the stimulation $(\boldsymbol{C}-\boldsymbol{E})$. $\boldsymbol{F}$, The mutants, unc-25 (F.1), unc-46 (F.2), and unc-49 (F.3), occasionally shrank without stimulation. Yellow and blue shaded areas in $\boldsymbol{B}-\boldsymbol{F}$ represent dorsoventral curvature (vertical axis is the length of animal from the head $(H)$ on the top to the tail (T) color bar is curvature $-10-10 \mathrm{~mm}^{-1}$, indifferent to dorsoventrality), along time (horizontal axis, common scale bar is $1 \mathrm{~s}$ ); black blocks indicate gap in tracking during the touch stimulation.

46 , and unc-49 during forward swimming were 271 (0.4\%), 3985 (14\%), $2086(3 \%)$, and 2965 (17\%), respectively; those during backward swimming were 138 (7\%), $1150(18 \%), 975(6 \%)$, and $1897(3 \%)$, respectively. Using these data, we calculated the linear regressions between undulation frequency and translocation speed for each of the free locomotion conditions, forward and backward crawling and swimming separately.

For the calcium imaging experiment, we included data analyzed from 11 focused and cell-identifiable videos of TOL15; 11 and 9 videos of CZ1632, TOL11 in $0.5 \%(\mathrm{w} / \mathrm{w})$ methylcellulose, respectively, and 12 and 9 videos in 3\% $(\mathrm{w} / \mathrm{w})$ methylcellulose, respectively. From these videos, we analyzed 27 and 24 ventral and dorsal body-wall muscles, respectively, in TOL15; 17 VD and 18 DD motoneurons in the high-frequency undulation and $26 \mathrm{VD}$ and 13 DD motoneurons in low frequency in CZ1632; and 32 VD and 15 DD motoneurons in the high-frequency undulation and $27 \mathrm{VD}$ and $14 \mathrm{DD}$ motoneurons in low frequency in TOL11.

Where possible, we used estimation statistics (Ho et al., 2019 ) to visualize our data in shared-control GardnerAltman plots, and to calculate the $p$ value by two-sided permutation $t$ test. The reported $p$ values are the likelihoods of observing the effect sizes, if the null hypothesis of zero difference is true. During bootstrapping, the control and test labels were reshuffled 5000 times for each permutation $p$ value.

\section{Code accessibility}

The code described in the paper is freely available online at https://bitbucket.org/leedswormlab/curve-worminhibition-paper. The code is available as Extended Data 1.

\section{Results}

\section{Both wild-type and GABA transmission mutants shrank in response to harsh touch to the head or tail}

C. elegans mutants defective in GABA transmission are known as "shrinkers" because of their distinct decrease in body length in response to a harsh touch to the head (Mclntire et al., 1993a,b; Schuske et al., 2004), to which wild-type animals respond with rapid backward motion. Because this phenotype has been described as a defect in backward locomotion, we first looked at the shrinking response of wild-type and GABA transmission mutant animals to harsh touch to the head, which induces rapid 
backward undulation in wild-type animals as well as the response to the tail touch to study the association of shrinking response to forward locomotion. To facilitate comparison to former studies, we used a simple stimulation method and touched the head or tail with a blunt firepolish glass probe. We chose three knock-out mutant strains in which hundreds to thousands of DNA base pairs had been deleted in the genes encoding the GABA synthesis enzyme glutamic acid decarboxylase (GAD, unc25, VC1433), vesicular transporter (unc-46, TM5916), and ionotropic GABA receptor (unc-49, TM5487), and compared them to the laboratory reference strain (wild-type, N2). We found that the shrinking response was common in wild-type and mutant animals following head or tail stimulation (Fig. 1A). To quantify the shrinking response of wild-type and the GABA transmission knock-out animals, we measured the change in body length for each strain and stimulation direction ( $n=9$ each) at four different time points (before, immediately after, 0.5 , and $2 \mathrm{~s}$ after stimuli), and normalized the body length to their value before stimuli (Fig. 2A; Extended Data Fig. 2-1). Immediately after a harsh touch to the head or tail, both wild-type and the GABA transmission knock-out animals decreased their body length: the body length of wild-type animals reduced to $94 \pm 2 \%$ and $95 \pm 3 \%$ (mean \pm SD), respectively; while the body length of GABA transmission knockouts was reduced to $90-91 \pm 2-4 \%$ and $91-92 \pm 3-4 \%$ immediately after harsh touch to the head or tail, respectively across the different strains. In addition, wildtype animals recovered to their prestimulus body length faster. At $2 \mathrm{~s}$ after the head or tail stimulation, the body length of wild-type animals reached $98 \pm 4 \%$ and $99 \pm 2 \%$ of the prestimulus body length; while that of GABA transmission knock-outs reached 92-94 $\pm 4-6 \%$ and $93-97 \pm 2-5 \%$, respectively, across the different strains (Fig. 2A; Extended Data Fig. 2-1). Moreover, GABA transmission knock-outs occasionally exhibited spontaneous shrinking (Fig. $1 F$ ). The brief shrinking of wild-type animals was only associated with vigorous escape response from strong aversive stimuli such as harsh touch and a strong chemical repellent $(97 \pm 1 \%$, $50 \mathrm{~mm} \mathrm{CuSO}_{4}$, on-food; Extended Data Fig. 2-1, table), but not with a weak chemical repellent $(99 \pm 1 \%, 0.1 \%$ SDS, off-food; Extended Data Fig. 2-1, table).

A possible explanation for the dorsoventral coactivation of body-wall musculature that causes body shrinkage in response to harsh touch is the simultaneous activation of all A-type and B-type cholinergic motoneurons by premotor interneurons that are associated with forward and backward locomotion. These distinct groups of interneurons activate either, DA and VA motoneurons during backward, or DB and VB motoneurons during forward locomotion (Haspel et al., 2010). Thus, we hypothesize that shrinking results from a short temporal overlap of activity of A-type and B-type cholinergic motoneurons. To test this hypothesis, we took advantage of developmental phenotypes that render mutants in a complementary pair of homeodomain transcription factors (UNC-4 and VAB-7), each lacking either A-type or B-type motoneurons. In unc-4 mutant animals (CB120),
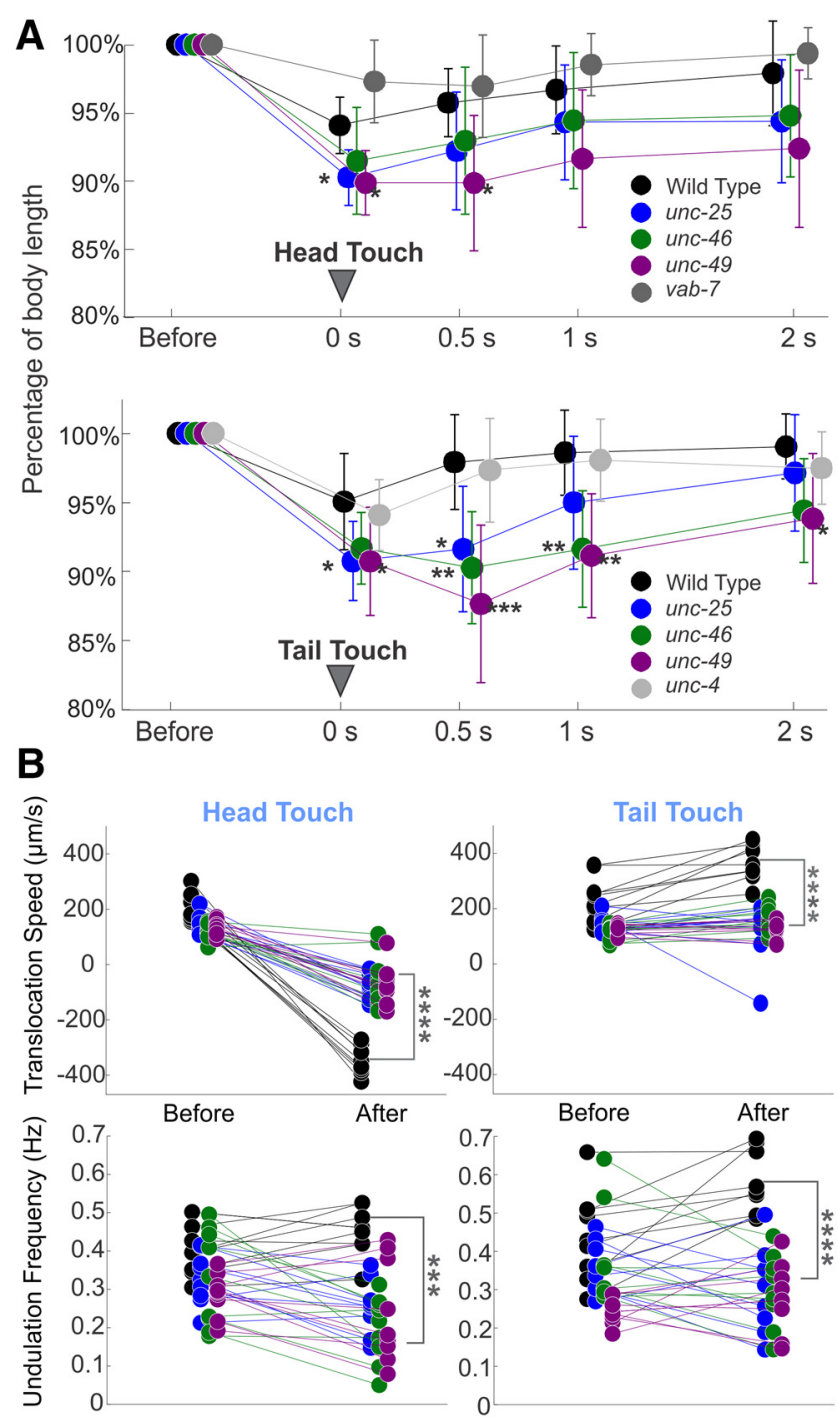

Figure 2. Both wild-type and GABA transmission knock-out animals shrank after harsh touch stimuli; wild-type animals shrank less, recovered sooner, and crawled away more rapidly than knock-out animals. $\boldsymbol{A}$, Wild-type and GABA transmission knock-out animals decreased their body length after harsh stimuli to the head or tail ( $0 \mathrm{~s})$. Compared with wild-type, GABA transmission mutants decreased their body length more and recovered more slowly $(0.5,1$, and $2 \mathrm{~s}$ after stimuli). The body length change of vab-7 and unc-4 were not statistically different from that of wild type. Body length is normalized to prestimulus value for each animal. See Extended Data Figure 2-1 for details. $\boldsymbol{B}$, Wild-type animals moved with higher mean undulation frequency and mean translocation speed during $5 \mathrm{~s}$ poststimulus compared with the $5 \mathrm{~s}$ prestimulus, while the mutants did not. Two unc-46 mutant animals did not change locomotion direction after harsh head touch, neither did one unc-25 mutant animal after harsh tail touch. In all plots, one-way ANOVA with Tukey's pairwise comparison to wild-type; ${ }^{*} p<0.05$, ${ }^{* \star} p<0.01,{ }^{* \star *} p<0.001,{ }^{\star \star \star *} p<0.0001$. Black circles: wild-type, blue: unc-25, green: unc-46, and purple: unc-49. See Extended Data Figures 2-2, 2-3 for details. 
cells destined to be A-type motoneurons differentiate as B-type motoneurons and receive input from forwardassociated premotor interneuron (Miller et al., 1992; Miller and Niemeyer, 1995). While it is unclear whether the misdifferentiated neurons fully function as B-type motoneurons, their function as A-type is impaired. Consequently, only their backward locomotion in uncoordinated. Hence, we tested whether unc-4 mutant animals shrink in response to harsh touch to the tail that induces an acceleration forward, toward their unaffected direction. Correspondingly, in vab-7 mutant animals (CB1562), cells destined to be DB motoneurons differentiate as DA motoneurons (Esmaeili et al., 2002). Consequently, only their forward locomotion is uncoordinated. Hence, we tested whether vab-7 mutant animals shrink in response to harsh touch to the head that induces an acceleration toward their unaffected direction. The harsh touch stimuli induced a brief reduction of body length in animals of both mutant strains (Fig. $2 A$ ) that was not different from that of the wild type (Extended Data Fig. 2-1, table). These results are inconsistent with the hypothesis of coactivation by premotor interneurons, suggesting instead that the shrinking phenotype is localized within the ventral nerve cord (VNC) motor circuit and not associated with directional change.

\section{GABA transmission knock-outs failed to escape as rapidly as wild-type after harsh touch stimulation}

After harsh touch stimulation and following the shrinking response, wild-type animals were able to escape at high-frequency and translocation speed, while the mutants moved away slowly (Fig. $1 A$, note location at $5 \mathrm{~s}$ ). We noticed that both wild-type and mutant animals suppress head swing during escape behavior. Suppression of head-swing was described as a part of the wild-type escape response and was found to be mediated by the tyraminergic system (Pirri et al., 2009). We measured the translocation speed and midbody undulation frequency as well as the maximal amplitude and primary wavelength, within $5 \mathrm{~s}$ before and $5 \mathrm{~s}$ after stimulation $(n=9$ video clips for each strain and stimulation location; Fig. 2B; Extended Data Figs. 2-2, 2-3). After harsh touch to the head or tail, wild-type animals escaped with significantly higher translocation speed and undulation frequency, while GABA transmission knock-outs had lower mean translocation speeds except for unc- 46 and unc- 49 after harsh tail touch, and lower mean undulation frequency, except for unc-49 after harsh tail touch. Compared with the locomotion within $5 \mathrm{~s}$ after the harsh head or tail touch of the wild-type, all the GABA transmission knock-outs had significantly lower translocation speed (one-way ANOVA with Tukey's test, $p<0.0001$ pairwise comparison to wild type) and undulation frequency ( $p<0.05$ pairwise comparison to wild-type; Fig. 2B; Extended Data Fig. 2-3). Wild-type and GABA transmission knock-outs exhibited larger amplitudes, but unchanged wavelengths after stimulation statistically (except for unc-46 after tail harsh touch; Fig. 2B; Extended Data Fig. 2-2). Note that to quantify the shrinking response, we applied harsh touch to sinusoidally forward crawling animals (both wild-type and mutants) and discarded assays that did not stand to this criterion. A possible bias from this selection underestimates the actual differences among strains.

\section{Defect in GABA transmission resulted in slow locomotion during free crawling and swimming}

Early descriptions of the locomotion defect of GABA transmission mutants and animals in which most GABAergic motoneurons have been laser-ablated emphasize the absence of backward locomotion (Mclntire et al., 1993b; Yanik et al., 2004). However, we noted that GABA transmission knock-out strains seem to crawl at low-frequency undulation in both directions, and their locomotion was more impaired during swimming in liquid (consistent with Boyle et al., 2012). Moreover, shrinking is not unique to backward locomotion but occurs in both mutants and wild-type animals regardless of direction. We recorded and compared unstimulated free crawling and swimming behaviors of wild-type and GABA transmission knock-out strains as well as an optogenetic strain (TOL12) in which GABAergic motoneurons can be inactivated acutely by light.

Crawling on the NGM agar surface without bacterial lawn, GABA transmission knock-out strains crawled slowly. The distribution of translocation speeds and frequencies of GABA transmission knock-outs during forward and backward crawling were significantly different from those of the wild type [Kolmogorov-Smirnov (KS) test, $p<0.0001$, for unc-25, unc-46, and unc-49]. The wild-type distribution of undulation frequency was bimodal for both forward and backward crawling. The undulation frequency during forward crawling fits two Gaussian distributions: a rapid distribution with 0.84 mixing proportion has a mean value of $0.47 \mathrm{~Hz}$, and a slow one with 0.16 mixing proportion has a mean value of $0.17 \mathrm{~Hz}$ (Fig. $3 A$ ). GABA transmission knock-out strains crawled at lower mean translocation speed and mean undulation frequency compared with the wild type (Fig. 3; Extended Data Fig. 31). Only the mean undulation frequency of VC1433 during forward crawling was not significantly lower than that of the wild type. The maximal amplitudes and primary wavelength of GABA transmission knock-outs were significantly smaller than those of the wild type.

During swimming, C. elegans undulates at higher frequencies, wavelengths, and amplitudes (Karbowski et al., 2006; Pierce-Shimomura et al., 2008; Berri et al., 2009; Fang-Yen et al., 2010; Butler et al., 2015). If the absence of GABA transmission impairs fast undulation, it should have a more severe effect on swimming behavior with a more distinct difference between mutants and wild type. We therefore recorded the swimming behavior of wildtype and GABA transmission knock-outs in NGM buffer. Wild-type animals alternated dorsal and ventral bends evenly with larger undulation amplitude and wavelength during swimming than during crawling (Fig. 4A1). In comparison, GABA transmission knock-outs undulated at a lower frequency with dorsoventral body alternation that was not as symmetric as that of the wild-type (Fig. 4A2). The distribution of translocation speeds and frequencies of GABA transmission knock-outs during forward and 
A
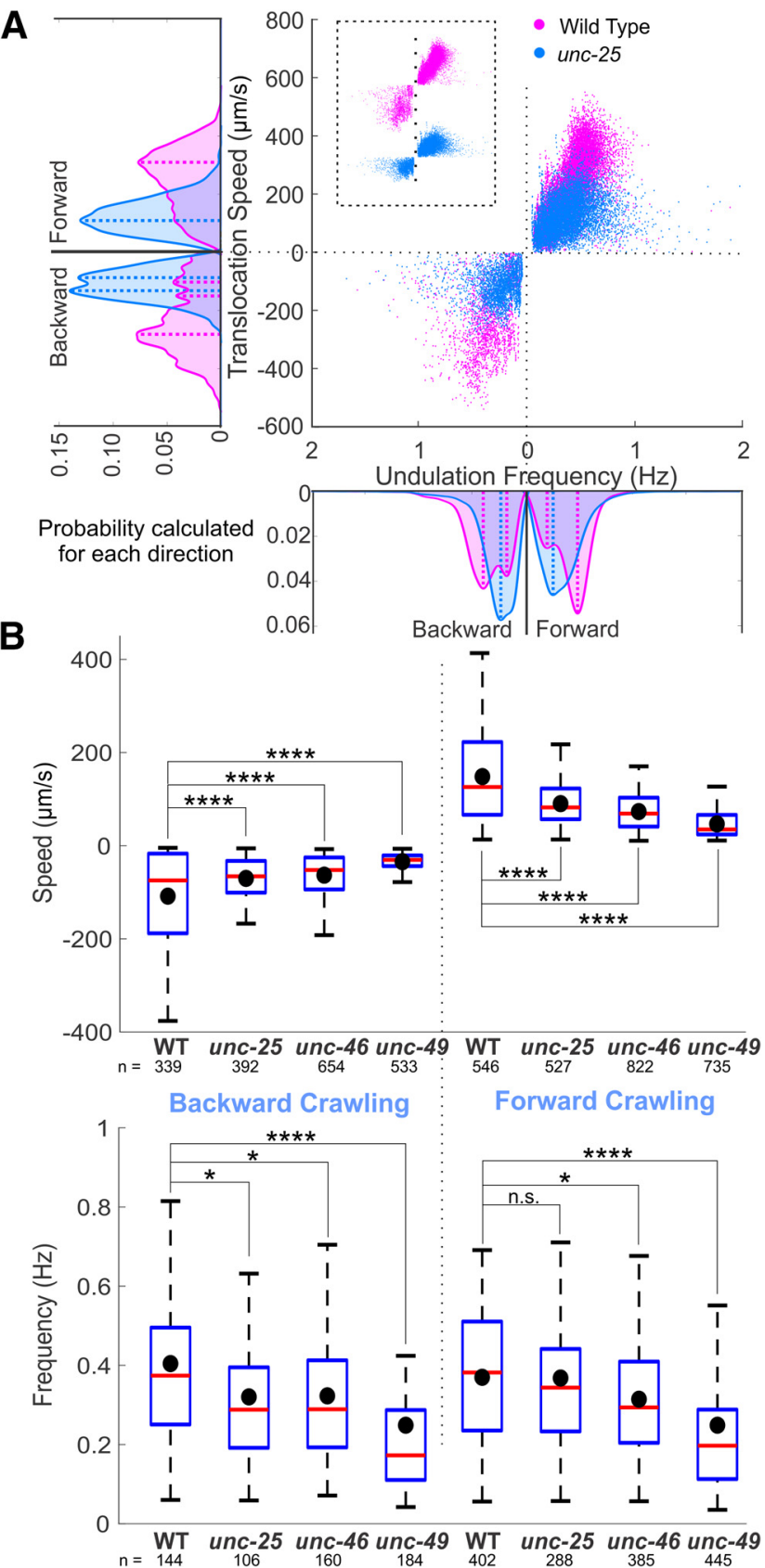

Figure 3. GABA transmission mutants crawled more slowly than wild type. $\boldsymbol{A}$, unc-25 knock-out animals crawled on agar surface with lower translocation speed and undulation frequency than wild type. Negative translocation speed and undulation frequency left (and right) of 0 represent backward (and forward) locomotion. Area plots show the probability of occurrence for each direction with dashed lines indicating the main peaks in the area plots. Probabilities were generated from histograms with bin size of $20 \mu \mathrm{m} / \mathrm{s}$ and $0.02 \mathrm{~Hz}$ translocation speed and undulation frequency, respectively. $\boldsymbol{B}$, Translocation speeds of all the GABA transmission knock-outs were significantly lower than that of wild-type (WT) during forward and backward crawling. The undulation frequencies of GABA transmission knock-outs were significantly lower than those of WT animals, except for that of unc-25 during forward crawling. Blue boxes: first to third quartiles of each dataset; red lines: medians; black continued

dots: mean values; whiskers: minimum and maximum, excluding outliers (beyond 1.5-fold interquartile range from middle $50 \%$ data). ANOVA with Tukey's pairwise comparison to WT, n.s. $p>0.05,{ }^{\star} p<0.05,{ }^{* \star \star} p<0.001,{ }^{* \star \star *} p<0.0001$. See Extended Data Figure 3-1 for details and Extended Data Figure 3-2 for shared-control Gardner-Altman plots.

backward swimming were significantly different from those of the wild type (KS test, $p<0.001$; for unc-25, unc46, and unc-49; Fig. 4B).

Indeed, GABA transmission knock-out strains swam at a significantly lower translocation speed and undulation frequency than the wild type (Fig. 4B,C; Extended Data Fig. 4-1). The maximal amplitudes and primary wavelength of GABA transmission knock-outs were significantly smaller than those of the wild type.

The GABA transmission knock-out animals are chronically impaired, and the effects we describe on locomotion could arise during development or because of compensation mechanisms. Therefore, we studied the locomotion of a transgenic strain (TOL12) that expresses Arch3 on the cellular membrane of GABAergic motoneurons, so they can be acutely inactivated by light. Arch3 is a light-sensitive proton pump that requires the cofactor ATR and causes hyperpolarization when exposed to lime-color light (Nagel et al., 2005; Okazaki et al., 2012). We fed transgenic animals with ATR for $24 \mathrm{~h}$ and tracked their swimming or crawling behaviors under infrared light (that does not activate Arch3) and then under lime-color light, which is the optimal activation wavelength of Arch3 (Mattis et al., 2011; Okazaki et al., 2012). To prevent desensitization of Arch3 after long exposure, we limited lime-color light to 1 min (Okazaki et al., 2012). We also tracked the locomotion behavior of transgenic animals that were not fed ATR as a negative control. When GABA transmission was inactivated acutely by hyperpolarizing GABAergic motoneurons, the frequency of undulation decreased, comparable to the effect of GABA transmission mutants. Swimming under lime-color light, the transgenic animals fed with ATR were slower and less coordinated, and their maximal amplitude and primary wavelength decreased, but quickly recovered when the optogenetic activation light was turned off (Fig. 5). Crawling on agar surface under lime-color light, the translocation speed of transgenic animals fed with ATR was significantly lower than that of the same animals under infrared light and that of the animals not fed ATR under lime-color light. Their undulation frequency was lower than that of the same animals under infrared light and that of the animals not fed ATR. Swimming in NGM buffer, their translocation speed was lower $(p<0.0001)$ than that of the same animals under infrared light and that of the animals not fed ATR. Their undulation frequency was lower $(p<0.0001)$ than that of the same animals under infrared light and that of the animals not fed ATR (Fig. 5D; Extended Data Fig. 5-1). In contrast with the swimming of GABA transmission mutants, some data points are as high frequency and fast as in control animals (compare Figs. $4 B$ and $5 C$ ), suggesting an incomplete optogenetic neuronal inactivation. 

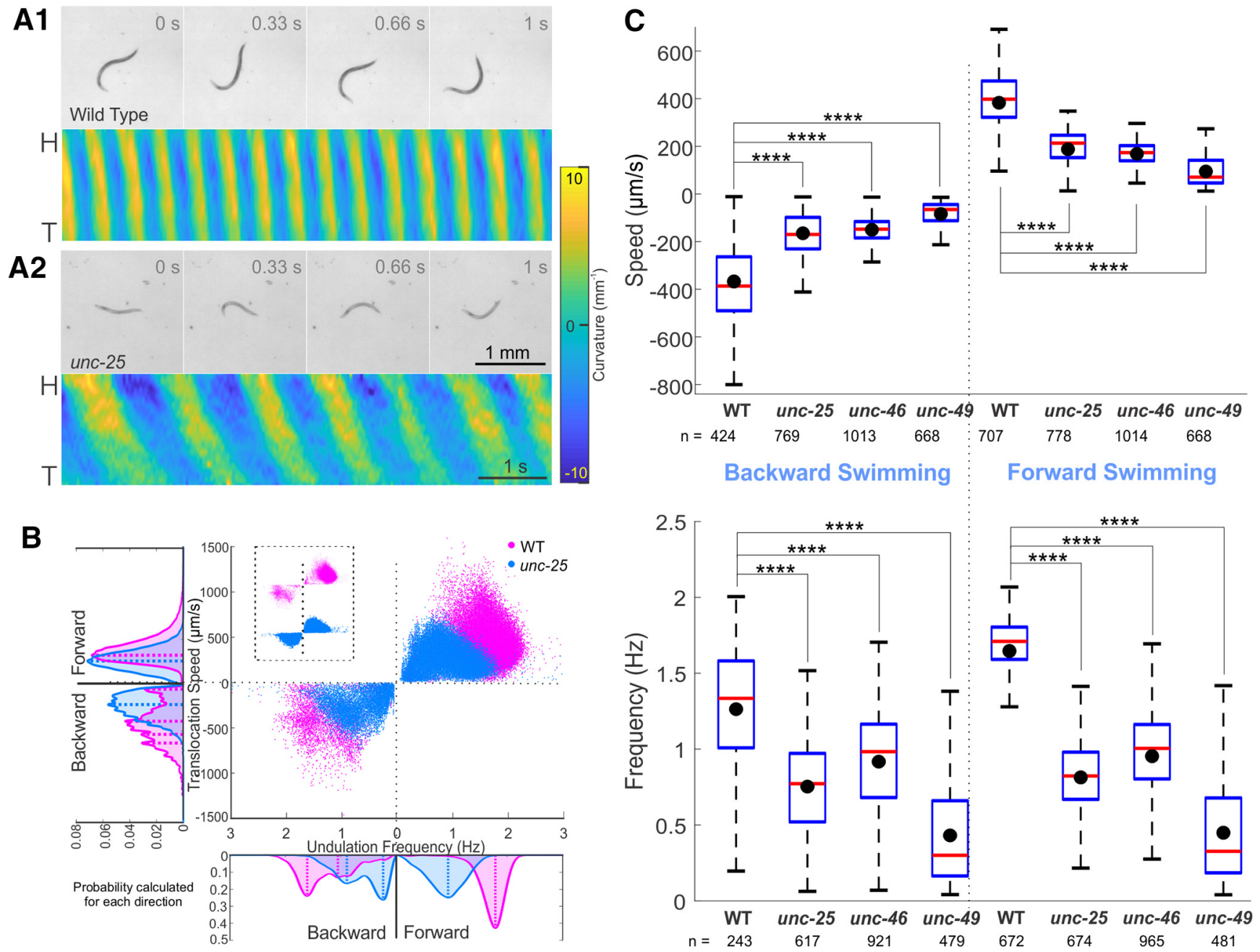

Figure 4. GABA transmission knock-outs swam more slowly than wild type. Compared with wild-type (A.1), unc-25 knock-out (A.2) in liquid swam with lower undulation frequency and translocation speed, as well as smaller undulation amplitude and wavelength. Video frames are $0.33 \mathrm{~s}$ apart, common scale bar is $1 \mathrm{~mm}$; yellow and blue shaded areas of kymograms represent dorsoventral curvature [vertical axis is the length of animal from the head $(\mathrm{H})$ on the top to the tail $(\mathrm{T})$ color bar is curvature $-10-10 \mathrm{~mm}^{-1}$, indifferent to dorsoventrality], along time (horizontal axis, scale bar is $1 \mathrm{~s}$ ). $\boldsymbol{B}$, unc-25 knock-out animals swam slower with lower translocation speed and undulation frequency than wild type. Area plots show the probability of occurrence for each direction with dashed lines indicating the main peaks in the area plots. Probabilities were generated from histograms with bin size of $24 \mu \mathrm{m} / \mathrm{s}$ and $0.05 \mathrm{~Hz}$ translation speed and undulation frequency, respectively. $\boldsymbol{C}$, Translocation speeds and undulation frequencies of all the GABA transmission knock-outs were significantly lower than those of wild-type (WT) during forward and backward swimming. Box plots as in Figure 3. ANOVA with Tukey's pairwise comparison to WT, ${ }^{* \star * \star} p<0.0001$. See Extended Data Figure $4-1$ for details and Extended Data Figure 4-2 for shared-control Gardner-Altman plots.

During free crawling or swimming, C. elegans exhibits a variety of behaviors from forward or backward almost-sinusoidal undulation, through $\omega$ and $\delta$ turns, direction changes, and pauses, to periods of quiescence (Stephens et al., 2008). When GABA transmission is impaired by knock-out mutations or optogenetic inactivation, C. elegans becomes less coordinated, and their undulation frequencies and translocation speed decrease. In order to compare the efficiency of harnessing undulation frequency to produce translocation speed in wild-type and mutant animals, we isolated and analyzed the almost-sinusoidal undulation. For all strains, in both directions of crawling and swimming, there were positive linear correlations between undulation frequency and translocation speed. Wild type translocated faster with the same undulation frequency compared with any of the GABA transmission knock-out strains (Fig. 6A,B).

\section{GABAergic motoneurons showed different activation patterns during low-frequency and high-frequency undulation}

In the absence of GABA transmission, animals did not move at rapid undulation frequency and fast translocation speed in either direction during free locomotion or in response to harsh touch stimulation to the head or tail. However, they were capable of slow dorsoventral undulation in both directions during free locomotion and after 


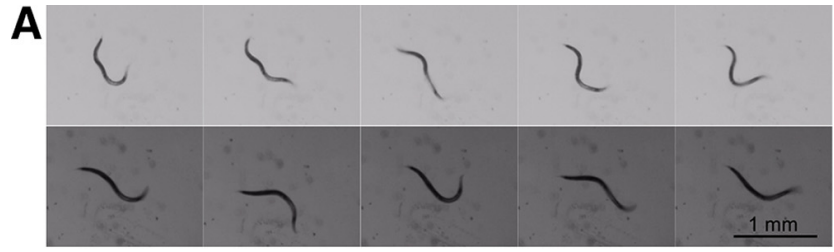

B

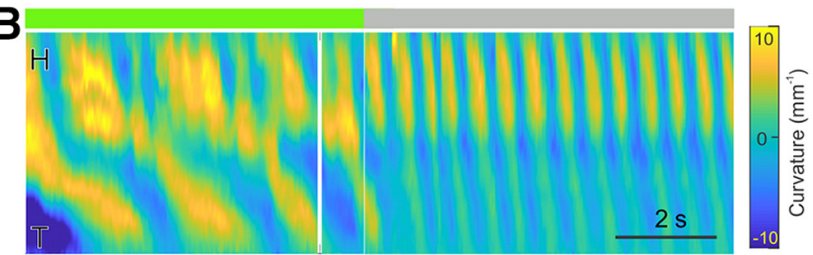

C
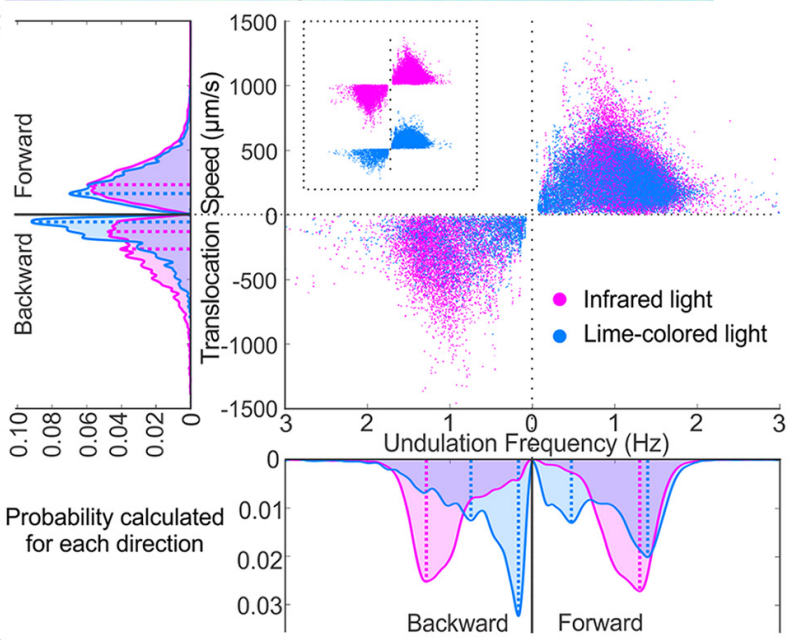

D
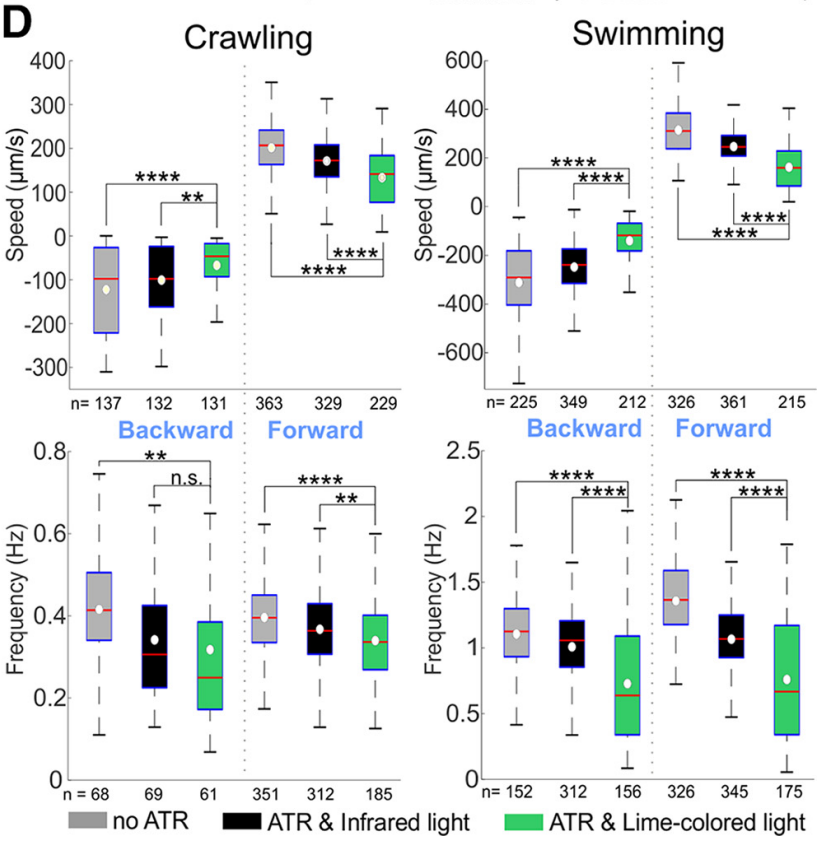

Figure 5. Acute optogenetic inactivation in GABAergic motoneurons induced slow locomotion during free swimming and crawling. $\boldsymbol{A}$, When GABAergic motoneurons were inactivated optogenetically (top framed and green line on top of kymogram) while swimming in liquid, undulation was slower and more uncoordinated. When the optogenetic inhibition was withdrawn (bottom frames and gray line on top of kymogram), the animal regained coordinated undulatory swimming and its undulation continued

frequency doubled from 0.5 to $1 \mathrm{~Hz}$. Postures shown at $0.33 \mathrm{~s}$ intervals; scale bar is $1 \mathrm{~mm}$; yellow and blue shaded areas of kymograms represent dorsoventral curvature (vertical axis is the length of animal from the head on the top to the tail, color bar is curvature $-10-10 \mathrm{~mm}^{-1}$, indifferent to dorsoventrality), along time (horizontal axis, scale bar is $1 \mathrm{~s}$ ). $\boldsymbol{B}$, The optogenetic strain fed with ATR swam slower when optogenetically inactivated (by lime-colored light) compared with the same animals under infrared light. Area plots show the probability of occurrence for each direction with dashed lines indicating the main peaks in the area plots. Probabilities were generated from histograms with bin size of $24 \mu \mathrm{m} / \mathrm{s}$ and $0.05 \mathrm{~Hz}$ translation speed and undulation frequency, respectively. $\boldsymbol{C}$, When GABAergic motoneurons were acutely inhibited (green boxes, ATR and lime-colored light), animals crawled and swam at a lower translocation speed and with lower undulation frequency compared with two negative controls, the same animal under infrared light (black boxes) and the same strain not fed ATR (gray boxes); except for backward-crawling undulation frequency that was not statistically different under lime-colored and infrared light. Boxplots similar to Figure 3. ANOVA with Tukey's pairwise comparison to the animal fed with ATR and under lime-colored light, n.s. $p>0.05,{ }^{\star \star} p<0.01$, ${ }^{\star \star \star \star \star ~}$ $p<0.0001$. See Extended Data Figure 5-1 for details and Extended Data Figures 5-2, 5-3 for shared-control Gardner-Altman plots.

stimulation. Moreover, when only sinusoidal undulation is considered, undulation frequency is a predictor of translocation speed in wild-type and mutant animals, with wild type exhibiting higher efficiency. Together, these results suggest that GABAergic motoneurons' contributions depend on undulation frequency and that they are necessary for high-frequency undulation.

To determine the contributions of GABAergic motoneurons during low-frequency and high-frequency undulation, we recorded their activity with a genetically encoded calcium sensor. To keep track of the locomotive phase and undulation frequency, we used a silicon microfluidic device with sinusoidal waveform channels designed to mimic the crawling path of wild-type animals (Fig. 7A; Lockery et al., 2008). Taking advantage of the predetermined shape of the channel and the published perimotor location of each motoneuron (Chen et al., 2006; Haspel and O'Donovan, 2011), we converted the soma position of GABAergic motoneurons during imaging to the locomotive phase of their neuromuscular junction to body-wall muscle. We assigned phases of the locomotion cycle to location in the channel according to the animal's dorsoventral bending and direction of movement. We define the peak of dorsal bending as $90^{\circ}$ and that of ventral bending as $270^{\circ}$, with other positions in the channel assigned accordingly. To manipulate the undulation frequency, we changed the viscosity of the fluid inside the channels with increasing concentration of methylcellulose (in NGM buffer) up to $3 \%(\mathrm{w} / \mathrm{w})$ methylcellulose (higher concentrations prevented animals from entering the channels). When animals crawled in $3 \%(\mathrm{w} / \mathrm{w})$ methylcellulose, the mean undulation frequency was $0.28 \pm 0.14 \mathrm{~Hz}$, which is close to the undulation frequencies of the GABA transmission knock-outs during forward crawling (0.32 \pm $0.18 \mathrm{~Hz}, p=0.89)$. Animals crawled faster in $0.5 \%(\mathrm{w} / \mathrm{w})$ 

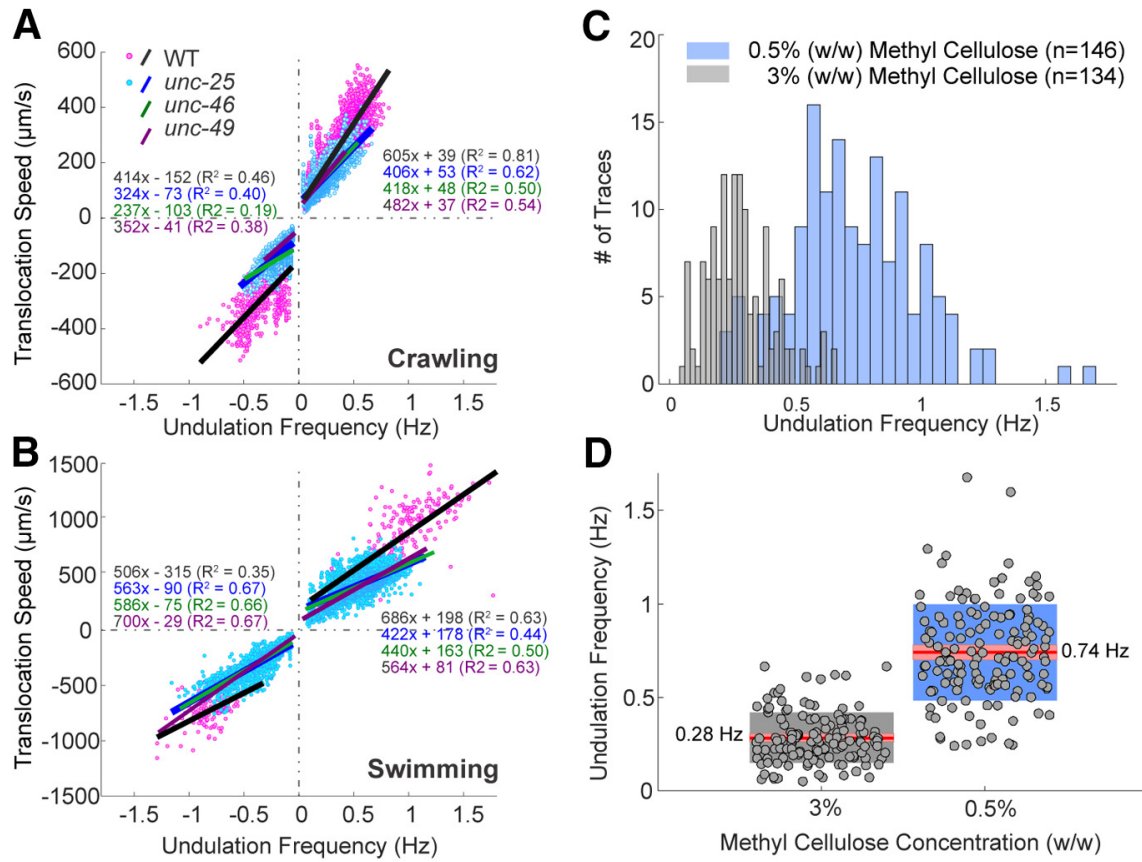

Figure 6. Undulation frequency correlated with translocation speed during free locomotion and undulation frequencies vary with the ambient viscosity in microfluidic waveform channels. $\boldsymbol{A}, \boldsymbol{B}$, Undulation frequency was positively correlated with translocation speed during crawling and swimming. Wild-type (magenta dots) and unc-25 (cyan dots) data are during episodes in which animals performed sinusoidal locomotion. Fitted linear regression lines of wild-type (black), unc-25 (blue), unc-46 (green), and unc-49 (purple) strains; coefficients of determination $\left(R^{2}\right)$ and equations are labeled with the same colors. $\boldsymbol{C}$, In waveform microfluidic devices, the animals can undulate in predetermined channels designed to restrict their path with corresponding sequences of postures. When the ambient viscosity was high (3\% w/w methylcellulose solution) wild-type animals undulated with lower undulation frequencies than in less viscous environment $(0.5 \% \mathrm{w} / \mathrm{w}$ methylcellulose solution). Frequency was measured by tracking the motion of neurons and muscle cells during calcium imaging. Sample size $(n)$ is the number of cells analyzed. $\boldsymbol{D}$, Same data as $\boldsymbol{C}$. The mean undulation frequency in $3 \%$ methylcellulose solution was $0.28 \pm 0.14$, and that in $0.5 \%$ solution was $0.74 \pm 0.26 \mathrm{~Hz}$. Red lines: mean values; pink boxes: $95 \%$ confidence intervals for the mean; gray and blue boxes: standard deviation; and gray dots: individual data points.

methylcellulose, with a mean undulation frequency of $0.74 \pm 0.26 \mathrm{~Hz}$ (Fig. 6C,D). We imaged calcium level changes in the cytoplasm of body-wall muscle cells with GCaMP2 (TOL15) in 1.5\% (w/w) methylcellulose and in the cytoplasm of GABAergic motoneurons with GCaMP6 (TOL11) in $0.5 \%$ and $3 \%(\mathrm{w} / \mathrm{w})$ methylcellulose. The average undulation frequency in $1.5 \%$ methylcellulose was $0.62 \pm 0.20 \mathrm{~Hz}$. Hence, regardless of methylcellulose concentration, we used $0.6 \mathrm{~Hz}$ as the cutoff to categorize data into low-frequency and high-frequency undulation. Because the animals rarely moved backward in the channels, we only collected and analyzed calcium imaging data for smooth forward locomotion.

The phase of the calcium imaging signal from body-wall muscle cells correlated to muscle contraction and bending. The signal increased in ventral muscle cells during ventral bending (around $270^{\circ}$; Fig. $7 B$ ) and in dorsal muscle cells during dorsal bending (around $90^{\circ}$ ), comparable to published calcium imaging signal during crawling on an agar surface (Butler et al., 2015). During high-frequency undulation, GABAergic motoneurons exhibited a sustained level of calcium signal with distinct troughs of phasic lower levels (Fig. 7C). VD motoneurons exhibited phasic low signal around ventral bending $\left(270^{\circ}\right)$, while DD motoneurons exhibited a corresponding phase of low signal around dorsal bending $\left(90^{\circ}\right)$. Both VD and DD had a phase of low signal around $180^{\circ}$, and only VD had a rise in calcium signal level between $270^{\circ}$ and $360^{\circ}$. In contrast, the calcium signal pattern in VD and DD during low-frequency undulation were similar to each other and not different from a recording from calcium-insensitive GFP expressed in GABAergic motoneurons: a wide increase in signal around $270^{\circ}$ that coincides with ventral bending. The increase in fluorescence intensity is likely because of convergence of fluorescence from neighboring motoneurons when the ventral nerve cord went through ventral bends.

Therefore, GABAergic motoneurons exhibited different patterns of activation during high-frequency and low-frequency undulation. In the high-frequency mode, VD and DD motoneurons have different inactive phases; in the low-frequency mode, VD and DD motoneurons showed similar wide inactive phases.

\section{Computational models tested three hypotheses for the role of inhibition in fast locomotion}

Based on the experimental results, we suggest five hypotheses to explain the role of inhibition in sustaining high-frequency locomotion. First, it has long been suggested that cross-inhibition of the opposing body-wall 


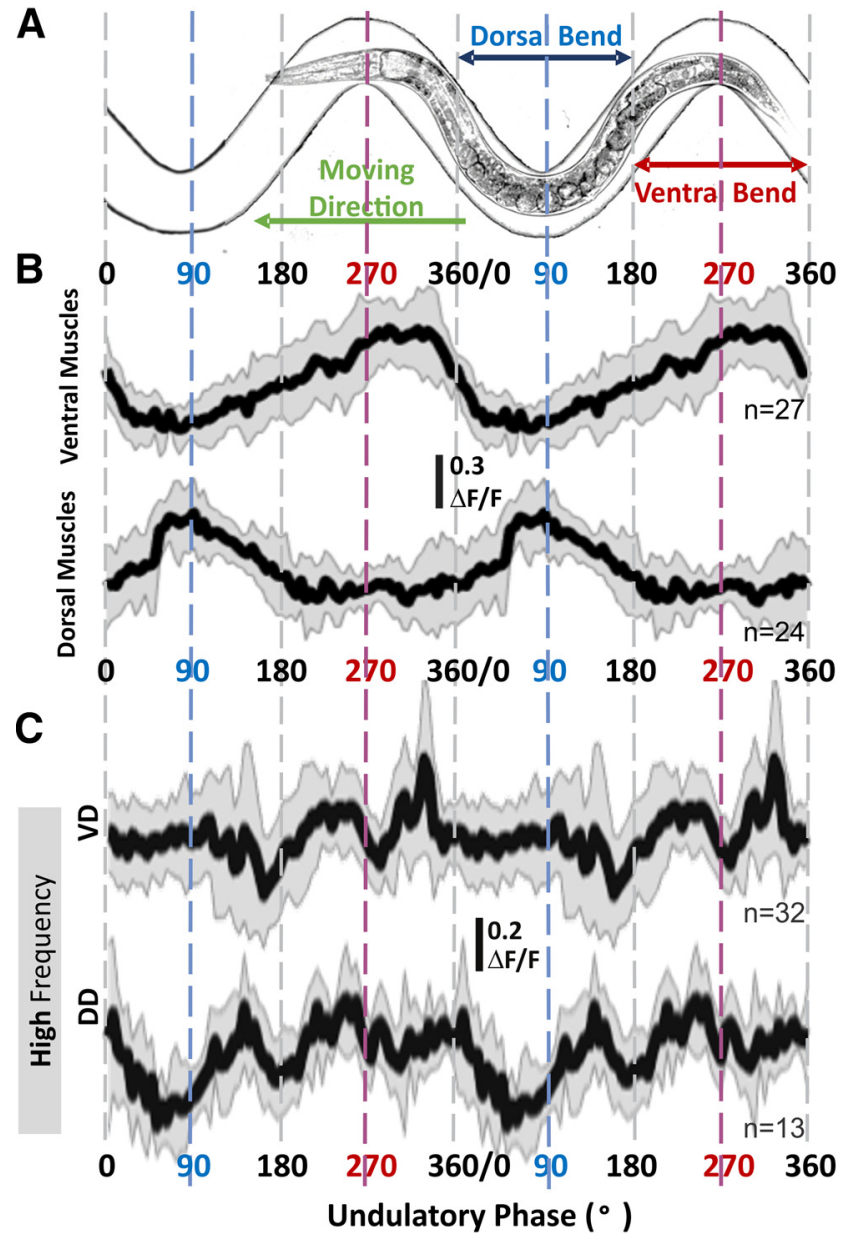

Figure 7. Calcium signal from GABAergic motoneurons related to locomotive phase and muscle activity only during high-frequency undulation. $\boldsymbol{A}$, Animals were free to move in sinusoidal microfluidic channels so that the phase of their undulatory cycle, including dorsal and ventral bends, is fixed and imposed by their spatial location. $\boldsymbol{B}$, Dorsal and ventral body-wall muscle cells were active during bending of the same side of the body (e.g., dorsal muscles were active during dorsal bend around $90^{\circ}$ ) regardless of undulation frequency. $\boldsymbol{C}$, During crawling at high-frequency undulation, VD showed two troughs around $180^{\circ}$ and $270^{\circ}$, and DD around $90^{\circ}$ and $180^{\circ}$; only VD showed a sharp increase around $300^{\circ}$. The sample size $(n)$ indicates the number of analyzed cells; solid lines and shaded areas in calcium traces are the mean and standard deviation. $\Delta \mathrm{F} / \mathrm{F}=$ $\left(F_{\text {top50\% }}-F_{\mathrm{BG}}\right) /\left(\Sigma\left(\mathrm{F}_{\text {top50\% }}-\mathrm{F}_{\mathrm{BG}}\right) / n\right)$. Changes in fluorescence levels and the perimotor-corrected locomotor phase were analyzed and calculated with a customized MATLAB program (Extended Data 1).

muscles increases the dorsoventral difference in muscle activation (McIntire, 1993a; Bryden and Cohen, 2008). Second, disinhibition of the innervated body-wall muscles increases muscle activation, particularly during the rising phase. Disinhibition, the excitatory effect of the removal of tonic inhibition, plays a role in control of other motor systems (Benjamin, et al., 2010), it does not exclude or require postinhibitory rebound. Third, inhibitory reset of VA and VB by the VD motoneurons allows higher locomotion frequency through phasic inhibition of ventral motoneurons (consistent with Boyle et al., 2012). Fourth, VD disinhibition of the VA and VB ventral cholinergic motoneurons amplifies, particularly during their rising phase. Fifth, reciprocal inhibition between VD and DD motoneurons stabilizes dorsoventral alternation.

From the experimental results, we found that vab-7 mutants (in which DB neurons differentiate as VA) and unc-4 mutants (in which DA and VA differentiate as DB and VB) were not different from the wild-type in their shrinking response to harsh head and tail touch, respectively. This suggests that the mechanism for the shrinking response is not caused by coactivation of forward and backward premotor interneurons (AVA and AVB), instead, it appears localized within the motor circuit. Furthermore, shrinking appears qualitatively similar in the forward and backward motor circuits. We therefore focused our computational models on forward locomotion. Our model results can be reproduced for backward locomotion by changing the direction of the propagating wave under feedforward control or by changing the direction of the proprioceptive field under feedback control (Denham et al., 2018). Our model results are only applied within the scope of the function of inhibition, and we do not assume that forward and backward circuits are driven by the same mechanism nor do we model the transitions between forward and backward locomotion.

There are two prevalent hypotheses to explain the undulatory body bends in $\mathrm{C}$. elegans that are not mutually exclusive: the proprioception (feedback-driven) hypothesis and the endogenous oscillator (feedforward-driven or CPG) hypothesis. Proprioception transduces body curvature to adjacent body segments, so that rhythmic movement propagates along the body; endogenous oscillators (CPG circuits) generate rhythmic movement at the head or tail of the animal, or along the body (Gjorgjieva et al., 2014). Although both proprioception and endogenous oscillators have been evidenced in C. elegans, their relative contributions during locomotion remain to be determined (Fouad et al., 2018; Gao et al., 2018; Xu et al., 2018). Similar to our approach here, previous models (Denham et al., 2018; Izquierdo and Beer, 2018) have investigated these regimes separately to parse their qualitative differences.

The models we used in this study are based on evidence for proprioceptive mechanisms (Wen et al., 2012) and for endogenous oscillators that could provide feedforward motor control (Gao et al., 2018). Using a proprioceptive model, we tested cross-inhibition, disinhibition, and inhibitory reset. Using a CPG-driven model, we tested muscle cross-inhibition and muscle disinhibition. Mechanisms of rhythm generation in the C. elegans nerve cord remain uncharacterized. We did not pursue or address in our models a possible role of GABAergic motoneurons in rhythm generation (Olivares et al., 2018), because if they play such a role, our experimental data demonstrate that it is not necessary for slow undulation. Furthermore, more detailed neural models would be required to test VD disinhibition of VA and VB motoneurons and reciprocal inhibition between VD and DD motoneurons (Fig. 8A). In addition, a consequence of modeling purely feedforward, CPG-driven motor activity is the absence of a mechanism for the modulation of frequency, e.g., in response to changes 
A

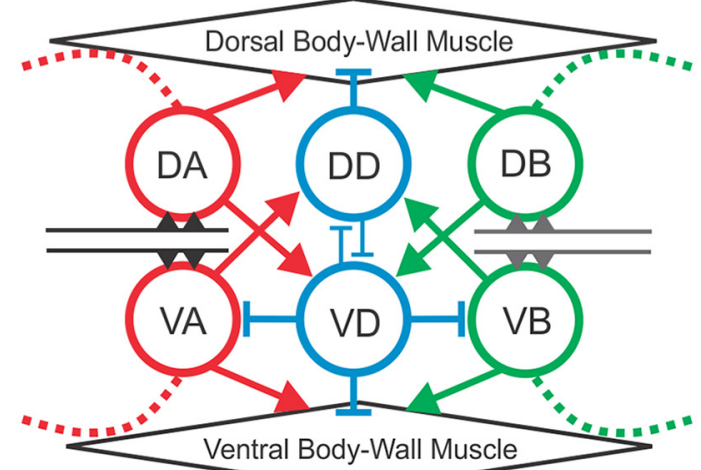

Forward

(1)

$\longrightarrow$ Excitatory Cholinergic Synapse

$\longrightarrow$ Inhibitory GABAergic Synapse

. ..... Stretch Receptor

Input from Premotor-interneuron

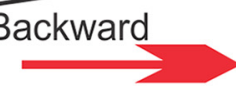

C

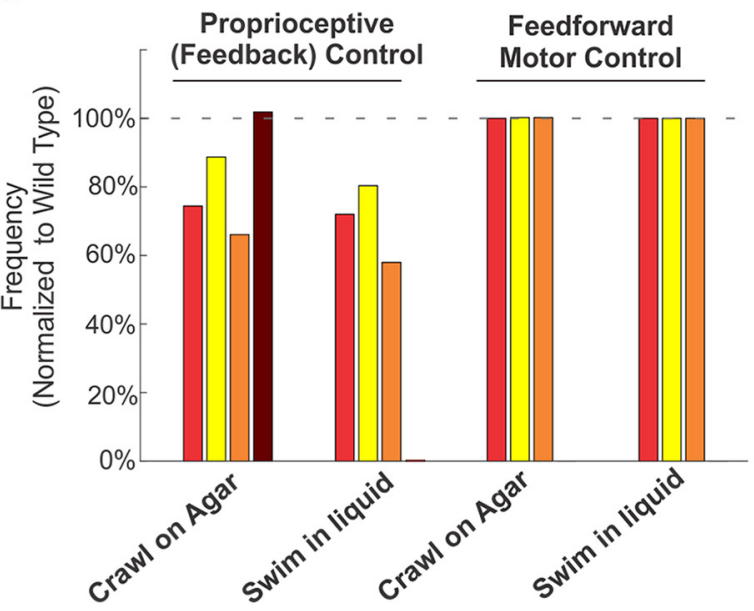

B

Proprioceptive

(Feedback) Control

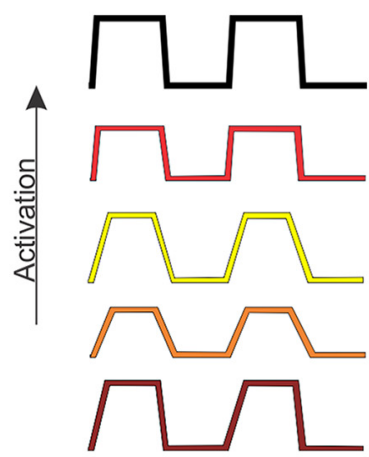

Feedforward

Motor Control

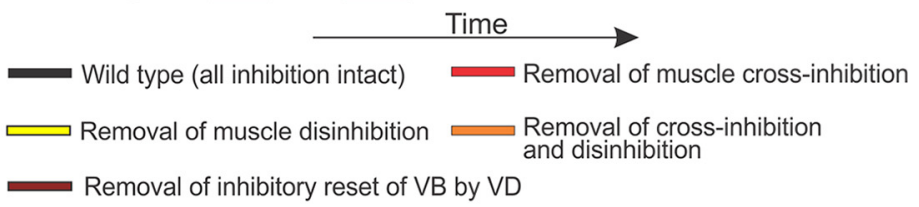

D

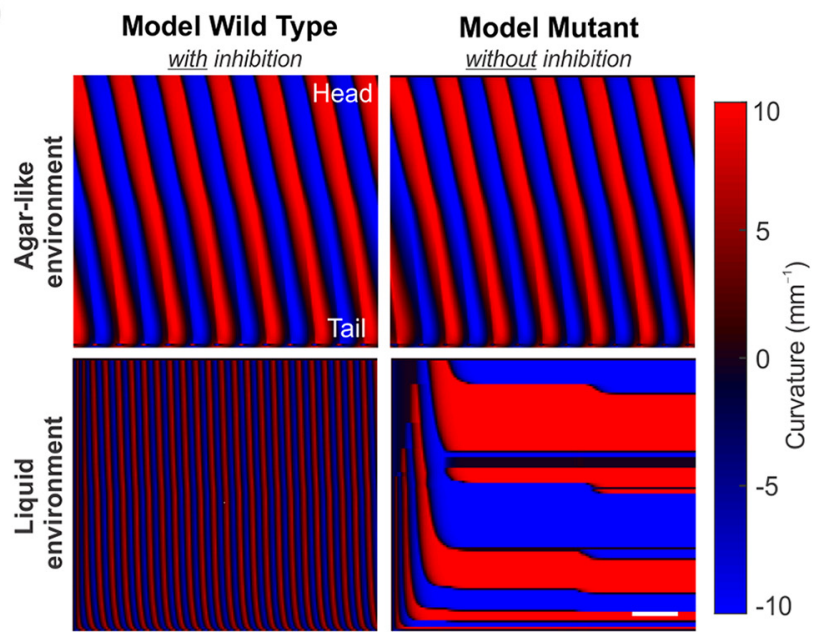

Figure 8. Integrated neuromechanical computational models test postulated roles of inhibition in proprioceptive (feedback) driven pattern generation, as compared with CPG (purely feedforward) models. $\boldsymbol{A}$, Repeating units of the neuromuscular circuit were used to model C. elegans locomotion control. Single-unit schematic motor circuit of the ventral nerve cord used in our proprioceptive model of locomotion. During forward locomotion, VB and VD (DB and DD) motoneurons innervate ventral (dorsal) muscles and VD motoneurons innervate VB motoneurons. During backward locomotion, A-type motoneurons take the place of B-type motoneurons. Postulated mechanosensation (dash lines) encode a proprioceptive signal by integrating the extent of bending over its receptive field. Suprathreshold proprioceptive currents trigger the activation of VB and DB motoneurons during forward locomotion (or VA and DA motoneurons during backward locomotion). The reciprocal synaptic connections between VD and DD motoneurons (blue thin lines) are not considered in the model. Adapted from Cohen and Denham (2019; their Fig. 1B). B, Schematic traces of muscle activation in the proprioceptive control model (left) and feedforward control model (right), under different model perturbations. Positive activation denotes dorsal muscle activation. By construction, dorsal and ventral activation are in exact antiphase. Three hypotheses for the role of inhibition (cross-inhibition of the opposing muscles; disinhibition of the innervated muscles; and inhibitory reset of VB by VD) were tested in proprioceptive models of motor control; postulated roles of muscle cross-inhibition and disinhibition were tested in feedforward models (parameters and parameter sweeps in Extended Data Figs. 8-1, 8-2, respectively). When muscle inhibition is disrupted, the amplitude or waveform of muscle input is modified from the model wild type (black). Shown are schematics of perturbations corresponding to hypothesis 1 (reduced amplitude, red), hypothesis 2 (slower/smoother waveform, yellow), hypotheses 1 and 2 combined (orange), and hypothesis 3 (removal of neural inhibition, brown, proprioceptive model only). $\boldsymbol{C}$, Relative frequency changes because of model perturbations. Under models of proprioceptive control, the undulation frequency is reduced by $\sim 10-35 \%$ relative to the wild type under a single perturbation to muscle inhibition, or up to $\sim 40 \%$ under the combined perturbation, suggesting that sufficient muscle activation amplitude and rapid activation onset are both required to sustain rapid undulation, during both crawling and swimming. Under proprioceptively driven locomotion, VB inhibition by VD serves to reset the neural rhythm during rapid undulation but has negligible effect in slow crawling-like motion. Bar plots with color scheme as in B depict the undulation frequency of model mutants normalized by the respective frequency of the model wild type. $\boldsymbol{D}$, Under a model of proprioceptive control, inhibitory reset is necessary for rapid swimming in a liquid environment (bottom), but not for slow crawling 
continued

on agar-like environments (top). In an agar-like environment, the elimination of VD-to-VB inhibition (right) has no effect. In a waterlike environment, elimination of this neural inhibition eliminates the rhythmic pattern altogether. Red and blue shaded areas represent dorsoventral curvature; vertical axis is the length of animal from the head $(\mathrm{H})$ on the top to the tail ( $\mathrm{T})$; color bar is ventral $(\mathrm{blue})$ to dorsal (red) curvature $-10-10 \mathrm{~mm}^{-1}$; along time (horizontal axis, common scale bar is $1 \mathrm{~s}$ ).

in environmental viscosity. For both models, we simulated locomotion in two environments (agar-like and liquid-like) but did not directly manipulate undulation frequency within a given environment. We simulated forward locomotion in agar-like and liquid-like environments under proprioceptive and CPG control. Then, we compared simulations of wildtype animals with simulations that incorporate perturbations of the neuromuscular input because of the omission of cross-inhibition, disinhibition, or both (Fig. 8B).

Under proprioceptive control, we find that all perturbations of muscle inhibition induced a substantial decrease in undulation frequency (Fig. 8C), whereas elimination of inhibitory reset resulted in a failure of rapid locomotion (Fig. 8C,D; Extended Data Fig. 8-2). Simulated mutants lacking cross-inhibition of the opposing muscles showed the largest reduction ( $25 \%$ and $28 \%$ of simulated wildtype frequency in agar-like and liquid-like environments, respectively, in response to a $20 \%$ change in muscle input) followed by simulated mutants lacking disinhibitory effect on the innervated muscle $(22 \%$ and $20 \%$ of wild-type frequency in agar-like and liquid-like environments, respectively, in response to a $20 \%$ change in muscle timescale). Applying both manipulations simultaneously decreased undulation frequency of the wild-type even further (by $34 \%$ and $42 \%$ in agar-like and liquid-like environments, respectively). Intuitively, for proprioceptive control, continuous activation of the antagonistic muscle during bending causes stiffness of muscles, slowing down bending, and in turn delaying the state switch of the adjacent motoneurons (i.e., the propagation of the undulatory wave). The same simulations also showed a decrease in overall locomotion speed, which was proportional to the frequency decrease in liquid, but disproportionately small in agar. In contrast, in our model of CPG control, muscle inhibition had no effect on the undulation frequency (Fig. 8C,D; Extended Data Fig. 8-2). We also found that removing cross-inhibition of model muscles results in reduced speed, whereas removing muscle disinhibition resulted in increased locomotion speeds, indicating that the propagation of smooth sinusoidal, rather than square, muscle activation wave is mechanically more efficient (Lighthill, 1960). Together, these results suggest that inhibition of GABAergic motoneurons influences undulation frequency primarily at the neuromuscular junctions. We note that additional frequency modulation mechanisms, which lie outside the scope of our model, cannot be ruled out under models of combined CPG and proprioceptive control.

We next tested the inhibitory resetting of VB by VD motoneurons. A neuromechanical model by Boyle et al. (2012) suggests that as a consequence of bistability of the A-type and B-type motoneurons (Liu et al., 2014), ventral and dorsal motoneurons may simultaneously occupy the same state, effectively pausing locomotion in a manner that is reminiscent of the shrinker phenotype. To resume healthy locomotion, Boyle et al. (2012) proposed that inhibition from VD to VB motoneurons acts as a neural reset mechanism, switching VB off to facilitate a dorsal bend. Simulations of Boyle et al.'s model show that this neural reset has little effect on slow locomotion (in an agar-like environment) but is required for coordinated undulation during fast locomotion (in a liquid-like environment). This result inspired our third hypothesis and the reimplementation of this mechanism in the current model (see Materials and Methods). We simulated nematodes lacking neural inhibition from VD to VB motoneurons in our proprioceptive model. The simulated mutant did not show any significant change in frequency in an agar-like environment (Figure 8C,D). However, when simulated in a liquid-like environment, these simulated mutants became severely uncoordinated and failed to make progress, consistent with Boyle et al. (2012). This result indicates a requirement for a neural inhibitory reset mechanism to sustain coordinated fast undulation, at least in the absence of additional backup mechanisms (such as CPG control).

To summarize, our model results suggest that under proprioceptively driven locomotion, muscle cross-inhibition enhances locomotion frequency and speed. The associated frequency-speed dependence is consistent with our experimental observations. In this model, cross-inhibition and disinhibition of the body-wall muscles supports high-frequency undulatory locomotion, whereas the neural inhibition within the motor circuit can stabilize high-frequency undulation.

\section{Discussion}

We investigated the role of inhibition in locomotion by analyzing the behavior of wild-type and mutant animals, recording neuronal and muscular activity in undulating intact animals, and by testing postulated roles of inhibition under different control paradigms with computational models. Both wild-type and GABA transmission mutants responded to harsh touch to the head or tail by shrinking, suggesting that dorsoventral coactivation is produced by the unimpaired nervous system. Impairment of GABA transmission genetically or optogenetically induced lower undulation frequency and translocation speed during crawling and swimming in both directions. GABAergic motoneurons' activity patterns were different during undulation at low and high frequencies. During low-frequency undulation, VD and DD showed a correlated activity pattern, while during high-frequency undulation, their activity alternated. We suggested five hypotheses for inhibitory mechanisms that support rapid undulation and tested three of them computationally. Our modeling results suggested that cross-inhibition and 
disinhibition of the body-wall muscles support rapid undulation. Finally, we suggest that the unimpaired locomotion circuit operates in two distinct modes to produce an undulatory motor program at low and high frequencies.

\section{Shrinking occurs in wild-type and GABA transmission mutants when initiating rapid locomotion in either direction}

The shrinking response, presumably from coactivation of the dorsoventral muscles, has been described in GABA transmission mutants (Mclntire et al., 1993a,b) and following laser ablation of GABAergic motoneurons (Yanik et al., 2004). Here, we show that shrinking commonly occurs in wild-type animals in response to harsh touch stimuli to the head or tail. In particular, we show that tail stimuli induce a rapid forward movement with change in frequency and speed but not direction. Furthermore, the shrinking response does not require the simultaneous contribution of cholinergic motoneurons associated with the forward and backward directions. Neurodevelopmental mutant animals (namely, vab-7 and unc-4) with impaired A-type or B-type cholinergic motoneurons, respectively, show a shrinking response after the harsh touch stimulation that induces their normal undulatory locomotion (e.g., harsh head touch to vab-7 induces wild type-like sinusoidal backward undulation). These results suggest that shrinking is a natural behavior and motor program that are produced by the unimpaired nervous system. Furthermore, they suggest that shrinking is not associated with backward locomotion or with a change in direction, but rather with the switch from slow to fast locomotion. The switch in locomotion speed could be a specific case of a more general phenomenon in which simultaneous contraction of antagonistic muscle occurs during the initiation of rapid behavior.

The shrinking phenotype was described in mutant animals (Mclntire et al., 1993a,b), but to date, it has not been reported in wild-type animals, probably because the latter resolves the coactivation and swiftly moves away from noxious stimuli. Video microscopy and frame-by-frame inspection are therefore required to capture the brief body shrinkage of wild-type animals. Morphologic measurement of the change in body length before and after harsh touch stimulation revealed that every animal, either wildtype or the GABA transmission knock-outs, shrank. It is the longer lasting shrinkage and lower translocation speed of GABA transmission mutants, which facilitates the observation of the shrinking phenotype.

\section{The distinct phenotype of the GABA transmission mutants is slow swimming}

GABA transmission mutants, colloquially named shrinkers have been described as defective in backward locomotion because they fail to produce wild type-like swift reversal following a harsh touch to the head (McIntire et al., 1993a,b; Schuske et al., 2004). A similar phenotype was described in animals after laser ablation (Yanik et al., 2004). However, we found that the shrinking phenotype is not related to the target of noxious stimuli or the direction of locomotion, as GABA transmission mutants shrink in response to harsh touch to the tail as well as the head. Moreover, wild-type animals also exhibit a shrinking phenotype, although smaller in magnitude and quicker to resolve. When suspended in liquid, GABA transmission mutants are uncoordinated and swim slowly with lower undulation frequency and translocation speed compared with those of the wild type. They exhibit a similar but less prominent phenotype when crawling on agar. Hence, slow uncoordinated swimming and exacerbated shrinking in response to aversive stimuli, are distinct, and possibly independent, phenotypes of GABA transmission mutants.

\section{GABA transmission is necessary for fast dorsoventral alternation}

During free crawling or swimming in either direction, an acute or chronic absence of GABA transmission leads to lower undulation frequency and translocation speed. The effect is more severe during swimming, which typically exhibits higher undulation frequency. Even following noxious touch stimuli that induce a rapid escape response in the wild type, GABA transmission mutants do not increase their undulation frequency and translocation speed and are slower than those of the wild type. These results deem GABA transmission necessary for fast locomotion. Similarly to our results, optogenetic activation of dopaminergic neurons induced a decrease in undulation frequency during crawling and swimming (Vidal-Gadea et al., 2011). That effect is, however, context dependent. Hence, decreased efficacy of GABA transmission could only partially explain the decrease in undulation frequency following increase in dopamine, and their effect could be mediated by different mechanisms.

The undulation frequency dictates translocation speed during sinusoidal crawling and swimming behaviors in wild-type and mutant animals. Correlations between undulation frequency and translocation speed were always positive. The slope of the linear regression was always steeper for the wild type, implying a more efficient translation of undulation frequency to translocation speed. This suggests a subtle contribution of inhibition to the motor program of slow locomotion because the data for mutant animals is of slower translocation speed. Kinematic parameters such as undulation amplitude and wavelength are weakly correlated to instantaneous translocation speed, although they may contribute to fast locomotion.

\section{GABAergic motoneurons exhibit dorsoventral alternating inactive phases that match the activation of their postsynaptic body-wall muscles only during high-frequency undulation}

Morphologically, GABAergic motoneurons synapse to the muscle arms of body-wall muscles (White et al., 1976, 1986), and their inhibitory effect on body-wall muscles was confirmed by laser ablation, muscle electrophysiology during bath application of GABA, and activation of GABAergic motoneurons optogenetically (Mclntire et al., 
1993b; Bamber et al., 2005; Gao and Zhen, 2011; Inoue et al., 2015). The neuronal activity of the GABAergic motoneurons during locomotion has not been reported and was presumed to be similar to that of their antagonistic muscle, as both are activated by the same excitatory motoneurons. However, they were recently suggested to be tonically active (Liu et al., 2020). Indeed, when we recorded the changes in calcium levels in GABAergic motoneurons during undulation, we found that they exhibited a baseline level of calcium signal interspaced by phases of lower levels of calcium signal and that the pattern of activity was frequency dependent. During high-frequency undulation, the calcium signal in VD and DD motoneurons decreased, respectively, when calcium signal levels in corresponding (ventral or dorsal) body-wall muscle cells increased (during contraction, at a phase of around $270^{\circ}$ of the locomotion cycle for ventral muscle, or $90^{\circ}$ for dorsal muscles (Fig. 7). These are possibly inactivity windows that allow postsynaptic muscles to escape from inhibition and be activated more sharply, or they could amplify muscle activity through disinhibition. In contrast, during low-frequency undulation, VD and DD exhibit correlated patterns of calcium signal changes with a wide increase in signal around $270^{\circ}$, coinciding with ventral bending. Hence, our calcium imaging of GABAergic motoneurons further supports their involvement in high-frequency undulation, as their activity patterns are different for low-frequency and high-frequency undulation. The underlying correlated pattern of the calcium signal during low-frequency undulation could be explained by the recent finding that the GABAergic motoneurons are mechanosensitive (Liu et al., 2020).

\section{Other possible inhibitory roles of GABAergic motoneurons in high-frequency undulation}

GABAergic motoneurons might play several inhibitory roles during high-frequency undulation. We tested three hypotheses for the neuronal mechanisms that underlie these roles using proprioceptive and feedforward motor control models: (1) cross-inhibition, in which GABAergic motoneurons inhibit the body-wall muscle opposing an activated muscle; (2) disinhibition of the innervated body-wall muscles, in which the release from inhibition enhances muscle activation; and (3) inhibitory reset of VA and VB, in which VD input terminates the locomotion cycle early and allows higher locomotion frequency (Boyle et al., 2012; Cohen and Sanders, 2014; Cohen and Denham, 2019). Our model results suggest that cross-inhibition and disinhibition of the body-wall muscles support high-frequency undulatory locomotion, whereas the neural inhibition within the motor circuit stabilizes such high-frequency undulation. Our model results are consistent with electrophysiological evidence for large GABA mediated current induced in body-wall muscle, but not in ventral A-motoneurons or Bmotoneurons when GABAergic motoneurons are activated optogenetically (Liu et al., 2009, 2020, Schultheis et al., 2011). In addition to the aforementioned roles of inhibition, there are other possible roles that we did not test because of experimental or computational model limitations. First, VD disinhibition of VA and VB, in which the activation of ventral cholinergic motoneurons is amplified, particularly during their rising phase. Second, reciprocal or nonreciprocal inhibition between DD and VD motoneurons, which might reinforce the antiphasic activity of GABAergic motoneurons during rapid locomotion. These effects of inhibition or disinhibition are not exclusive in sustaining the fast dorsoventral body-wall muscle alternation. Cross-inhibition and disinhibition of muscles enhance the activation-relaxation alternation of dorsoventral muscles, and neural inhibition provides a reset to allow rapid undulation patterns, while providing redundancy to support slow oscillations.

\section{Two modes of locomotion}

We have demonstrated that the inhibitory motoneurons of the locomotion circuit are necessary only for rapid undulation and that their activity patterns are different for rapid and slow undulation. The bimodal distributions of frequency and translocation speed during wild-type crawling in both directions (Fig. $3 A$ ) indicate two distinct modes of locomotion: slow and fast. The frequency-dependent activity pattern of inhibitory motoneurons, the brief shrinking behavior of wild-type animals when initiating an escape response, and the absence of rapid undulation when inhibition is inactive all suggest a role for inhibition in the unimpaired nervous system. Together, these results suggest that the locomotion circuit produces an undulatory motor program by two distinct modes of operation: low-frequency undulation that do not require inhibition and high-frequency undulation that require inhibition and in which GABAergic motoneurons exhibit an alternating activity pattern. Note that the two modes operate in different environment viscosity. Accordingly, animals that lack GABA transmission cannot swim or crawl as rapidly as wild-type animals, but they can swim at higher frequency than that exhibited by crawling wild-type animals (compare Figs. $3 A$ and $4 B$ ). The ranges of frequencies and speeds overlap between the two modes and are each likely affected by sensory and mechanical feedback, and possibly by modulation of a feedforward motor pattern.

\section{A fundamental role for inhibition in rapid locomotion}

Involvement of neuronal inhibition in the control of frequency and speed of behavior seems to be a fundamental mechanistic principle among locomotor circuits. In Drosophila larvae, a set of inhibitory local interneurons, termed period-positive median segmental interneurons (PMSIs) control the frequency and speed of peristaltic locomotion by limiting the duration of motoneuronal bursting (Kohsaka et al., 2014). In adult Drosophila, similar yet unidentified inhibitory premotor input to leg motoneurons is associated with walking speed and their inactivation reveals an underlying slower walking speed (Gowda et al., 2018). In contrast, a lesion of the crossed glycinergic fibers or blocking glycinergic transmission in the lamprey spinal cord increase fictive burst frequency (Grillner, 2003). In the Xenopus tadpole high-frequency swimming is correlated to both strong background excitation and strong phasic inhibition from the inhibitory interneuron pools alN and cIN, while weakening inhibition 
from dINs slows swimming down (Li and Moult, 2012). In zebrafish commissural local (CoLos) interneurons are recruited only during a rapid escape behavior (Liao and Fetcho, 2008; Satou et al., 2009). The glycinergic CoLos receive gap junctions from the Mauthner cell axon and inhibit the contralateral motoneurons as well as contralateral $\mathrm{CiD}$ and CoLo interneurons, thus inhibiting the driving force for contralateral muscle and disinhibiting the ipsilateral neuronal populations (Satou et al., 2009). In addition, ventrally located commissural bifurcating longitudinal (CoBLs) interneurons are only active at higher swimming frequencies (Liao and Fetcho, 2008). In the mammalian spinal cord (mostly studied in the mouse and cat), V1 neurons, a class of local circuit inhibitory interneurons, are involved in the regulation of leg motoneuron bursting and step cycle duration to control the stepping frequency and speed of walking (Gosgnach et al., 2006). Genetic ablation or silencing of V1 neurons induces a marked decrease in locomotor frequency with no apparent effect of flexor-extensor coordination, while blocking synaptic output of both $\mathrm{V} 1$ and $\mathrm{V} 2 \mathrm{~b}$ neuronal populations, flexor-extensor pattern becomes synchronous (Zhang et al., 2014). In contrast, $\mathrm{V}_{\mathrm{D}}$ inhibitory commissural interneurons coordinate left-right alternation used in slow (walk) rather than fast (trot) gaits (Bellardita and Kiehn, 2015; Kiehn, 2016). Finally, a truncation mutation in the transcription factor gene dmrt3 gives Icelandic horses their typical tölting gait (characterized by the legs on the same body side moving forwards or backwards in synchrony), as well as pacing and ambling gaits in other breeds (Andersson et al., 2012). The protein DMRT3 is expressed in interneurons in the spinal cord, including inhibitory commissural neurons, suggesting that they are involved in gait and locomotion speed.

The frequency-supporting inhibitory inputs are mediated in insects and vertebrates by premotor interneurons that we suggest are analogous to motoneurons in the ventral nerve cord of $C$. elegans (Haspel et al., 2020). In this framework, the five pairs of nematode locomotion interneurons are analogous to descending interneurons (e.g., corticospinal or reticulospinal), whereas nematode motoneurons are analogous to spinal interneurons, integrating sensory and descending inputs, while generating and coordinating motor programs via their interconnectivity. It follows that nematode muscle arms are analogous to spinal motoneurons and each nematode muscle cell constitutes a motor unit. Accordingly, some C. elegans motoneurons are dedicated to a direction of locomotion (Haspel et al., 2010), while the muscle arms serve as the "final common path" (Sherrington, 1906). Furthermore, in C. elegans a small number of identifiable neurons produce locomotion, whose activity can be recorded and controlled noninvasively with optical and genetic tools in freely moving animals (Fouad et al., 2018; Gao et al., 2018; Tolstenkov et al., 2018; Haspel et al., 2020). Hence, further study of the involvement of inhibition in rapid locomotion in $C$. elegans will likely provide useful comparative insight (Büschges et al., 2011; Katz and Hale, 2017) for understanding the functional role, and possibly the evolutionary origin, of premotor inhibition in locomotory circuitry at large.

\section{References}

Ahringer J (2006) Reverse genetics. WormBook, April 6, 2006.

Alon U (2007) Network motifs: theory and experimental approaches. Nat Rev Genet 8:450-461.

Altun ZF, Hall DH (2009) Muscle system, somatic muscle. WormAtlas, May 31, 2013.

Andersson LS, Larhammar M, Memic F, Wootz H, Schwochow D, Rubin CJ, Patra K, Arnason T, Wellbring L, Hjälm G, Imsland F, Petersen JL, McCue ME, Mickelson JR, Cothran G, Ahituv N, Roepstorff L, Mikko S, Vallstedt A, Lindgren G, et al. (2012) Mutations in DMRT3 affect locomotion in horses and spinal circuit function in mice. Nature 488:642-646.

Bamber BA, Richmond JE, Otto JF, Jorgensen EM (2005) The composition of the GABA receptor at the Caenorhabditis elegans neuromuscular junction. Br J Pharmacol 144:502-509.

Bellardita C, Kiehn O (2015) Phenotypic characterization of speedassociated gait changes in mice reveals modular organization of locomotor networks. Curr Biol 25:1426-1436.

Benjamin PR, Staras K, Kemenes G (2010) What roles do tonic inhibition and disinhibition play in the control of motor programs? Front Behav Neurosci 4:30.

Berri S, Boyle JH, Tassieri M, Hope IA, Cohen N (2009) Forward locomotion of the nematode $C$. elegans is achieved through modulation of a single gait. HFSP J 3:186-193.

Boyle JH, Berri S, Cohen N (2012) Gait modulation in C. elegans: an integrated neuromechanical model. Front Comput Neurosci 6:10.

Brenner S (1974) The genetics of Caenorhabditis elegans. Genetics 77:71-94.

Brown TG (1912) The factors in rhythmic activity of the nervous system. Proc Royal Soc B 85:278-289.

Bryden J, Cohen N (2008) Neural control of Caenorhabditis elegans forward locomotion: the role of sensory feedback. Biol Cybern 98:339-351.

Bucher D, Haspel G, Golowasch J, Nadim F (2015) Central pattern generators. In: Encyclopedia of Life Sciences. Hoboken: John Wiley \& Sons.

Büschges A, Scholz H, El Manira A (2011) New moves in motor control. Curr Biol 21:R513-R524.

Butler VJ, Branicky R, Yemini E, Liewald JF, Gottschalk A, Kerr RA, Chklovskii DB, Schafer WR (2015) A consistent muscle activation strategy underlies crawling and swimming in Caenorhabditis elegans. J R Soc Interface 12:20140963.

Chalfie M, Sulston JE, White JG, Southgate E, Thomson JN, Brenner $S$ (1985) The neural circuit for touch sensitivity in Caenorhabditis elegans. J Neurosci 5:956-964.

Chen BL, Hall DH, Chklovskii DB (2006) Wiring optimization can relate neuronal structure and function. Proc Natl Acad Sci USA 103:4723-4728.

Chow BY, Han X, Dobry AS, Qian X, Chuong AS, Li M, Li M, Henninger MA, Belfort GM, Lin Y, Monahan PE, Boyden ES (2010) High-performance genetically targetable optical neural silencing by light-driven proton pumps. Nature 463:98-102.

Cohen N, Boyle JH (2010) Swimming at low Reynolds number: a beginners guide to undulatory locomotion. Contemp Phys 51:103123.

Cohen N, Sanders T (2014) Nematode locomotion: dissecting the neuronal-environmental loop. Curr Opin Neurobiol 25:99-106.

Cohen N, Ranner T (2017) A new computational method for a model of $C$. elegans biomechanics: insights into elasticity and locomotion performance. arXiv 1703.04988.

Cohen N, Denham JE (2019) Whole animal modeling: piecing together nematode locomotion. Curr Opin Syst Biol 13:150-160.

Corsi AK, Wightman B, Chalfie M (2015) A transparent window into biology: a primer on Caenorhabditis elegans. WormBook, June 18, 2015. 
Cregg JM, Chu KA, Dick TE, Landmesser LT, Silver J (2017) Phasic inhibition as a mechanism for generation of rapid respiratory rhythms. Proc Natl Acad Sci USA 114:12815-12820.

Denham JE, Ranner T, Cohen N (2018) Signatures of proprioceptive control in Caenorhabditis elegans locomotion. Philos Trans R Soc Lond B 373:20180208.

Emmons SW (2015) The beginning of connectomics: a commentary on White et al. (1986) 'The structure of the nervous system of the nematode Caenorhabditis elegans'. Philos Trans R Soc Lond B Biol Sci 370:20140309.

Esmaeili B, Ross JM, Neades C, Miller DM 3rd, Ahringer J (2002) The C. elegans even-skipped homologue, vab-7, specifies DB motoneurone identity and axon trajectory. Development 129:853-862.

Ezcurra M, Tanizawa Y, Swoboda P, Schafer WR (2011) Food sensitizes $C$. elegans avoidance behaviours through acute dopamine signalling. EMBO J 30:1110-1122.

Fang-Yen C, Wyart M, Xie J, Kawai R, Kodger T, Chen S, Wen Q, Samuel AD (2010) Biomechanical analysis of gait adaptation in the nematode Caenorhabditis elegans. Proc Natl Acad Sci USA 107:20323-20328.

Forbes A (1912) Reflex rhythm induced by concurrent excitation and inhibition. Proc Royal Soc B 85:289-298.

Fouad AD, Teng S, Mark JR, Liu A, Alvarez-Illera P, Ji H, Du A, Bhirgoo PD, Cornblath E, Guan SA, Fang-Yen C (2018) Distributed rhythm generators underlie Caenorhabditis elegans forward locomotion. Elife 7:e29913.

Friesen WO (1994) Reciprocal inhibition: a mechanism underlying oscillatory animal movements. Neurosci Biobehav Rev 18:547-553.

Gao S, Zhen M (2011) Action potentials drive body wall muscle contractions in Caenorhabditis elegans. Proc Natl Acad Sci USA 108:2557-2562.

Gao S, Guan SA, Fouad AD, Meng J, Kawano T, Huang YC, Li Y, Alcaire S, Hung W, Lu Y, Qi YB, Jin Y, Alkema M, Fang-Yen C, Zhen M (2018) Excitatory motor neurons are local oscillators for backward locomotion. Elife 7:e29915.

Garcia D (2010) Robust smoothing of gridded data in one and higher dimensions with missing values. Comput Stat Data Anal 54:11671178.

Gershenson C, Helbing D (2015) When slower is faster. Complexity 21:9-15.

Gjorgjieva J, Biron D, Haspel G (2014) Neurobiology of Caenorhabditis elegans locomotion: where do we stand? Bioscience 64:476-486.

Gosgnach S, Lanuza GM, Butt SJ, Saueressig H, Zhang Y, Velasquez T, Riethmacher D, Callaway EM, Kiehn O, Goulding M (2006) V1 spinal neurons regulate the speed of vertebrate locomotor outputs. Nature 440:215-219.

Gowda SB, Paranjpe PD, Reddy OV, Thiagarajan D, Palliyil S, Reichert H, VijayRaghavan K (2018) GABAergic inhibition of leg motoneurons is required for normal walking behavior in freely moving Drosophila. Proc Natl Acad Sci USA 115:E2115-E2124.

Grillner S (2003) The motor infrastructure: from ion channels to neuronal networks. Nat Rev Neurosci 4:573-586.

Grillner S (2006) Biological pattern generation: the cellular and computational logic of networks in motion. Neuron 52:751-766.

Hart AC (2006) Behavior. WormBook, July 3, 2006.

Haspel G, O'Donovan MJ (2011) A perimotor framework reveals functional segmentation in the motoneuronal network controlling locomotion in Caenorhabditis elegans. J Neurosci 31:1461114623.

Haspel G, O'Donovan MJ, Hart AC (2010) Motoneurons dedicated to either forward or backward locomotion in the nematode Caenorhabditis elegans. J Neurosci 30:11151-11156.

Haspel G, Deng L, Harreguy MB, Tanvir Z (2020) Elegantly. In: The neural control of movement (Whelan PJ, Sharples SA, eds). Cambridge: Elsevier Academic Press.

Hilliard MA, Bergamasco C, Arbucci S, Plasterk RH, Bazzicalupo P (2004) Worms taste bitter: ASH neurons, QUI-1, GPA-3 and ODR3 mediate quinine avoidance in Caenorhabditis elegans. EMBO J 23:1101-1111.
Ho J, Tumkaya T, Aryal S, Choi H, Claridge-Chang A (2019) Moving beyond $P$ values: data analysis with estimation graphics. Nat Methods 16:565-566.

Inoue M, Takeuchi A, Horigane S, Ohkura M, Gengyo-Ando K, Fujii H, Kamijo S, Takemoto-Kimura S, Kano M, Nakai J, Kitamura K, Bito H (2015) Rational design of a high-affinity, fast, red calcium indicator R-CaMP2. Nat Methods 12:64-70.

Izquierdo EJ, Beer RD (2018) From head to tail: a neuromechanical model of forward locomotion in Caenorhabditis elegans. Philos Trans R Soc Lond B 373:20170374.

Javer A, Currie M, Lee CW, Hokanson J, Li K, Martineau CN, Yemini E, Grundy LJ, Li C, Ch'ng Q, Schafer WR, Nollen EAA, Kerr R, Brown AEX (2018) An open-source platform for analyzing and sharing worm-behavior data. Nat Methods 15:645-646.

Katz PS, Hale ME (2017) Evolution of motor systems. In: Neurobiology of motor control: fundamental concepts and new directions (Hooper SL, Büschges A, eds), pp 135-176. Hoboken: John Wiley \& Sons.

Karbowski J, Cronin CJ, Seah A, Mendel JE, Cleary D, Sternberg PW (2006) Conservation rules, their breakdown, and optimality in Caenorhabditis sinusoidal locomotion. J Theor Biol 242:652-669.

Kiehn O (2011) Development and functional organization of spinal locomotor circuits. Curr Opin Neurobiol 21:100-109.

Kiehn O (2016) Decoding the organization of spinal circuits that control locomotion. Nat Rev Neurosci 17:224-238.

Kohsaka H, Takasu E, Morimoto T, Nose A (2014) A group of segmental premotor interneurons regulates the speed of axial locomotion in Drosophila larvae. Curr Biol 24:2632-2642.

Li WC, Moult PR (2012) The control of locomotor frequency by excitation and inhibition. J Neurosci 32:6220-6230.

Liao JC, Fetcho JR (2008) Shared versus specialized glycinergic spinal interneurons in axial motor circuits of larval zebrafish. J Neurosci 28:12982-12992.

Lighthill M (1960) Note on the swimming of slender fish. J Fluid Mech 9:305-317.

Liu P, Chen B, Wang ZW (2014) SLO-2 potassium channel is an important regulator of neurotransmitter release in Caenorhabditis elegans. Nat Commun 5:5155.

Liu P, Chen B, Wang ZW (2020) GABAergic motor neurons bias locomotor decision-making in C. elegans. Nat Comm 11:1-9.

Liu Q, Hollopeter G, Jorgensen EM (2009) Graded synaptic transmission at the Caenorhabditis elegans neuromuscular junction. Proc Natl Acad Sci USA 106:10823-10828.

Lockery SR, Lawton KJ, Doll JC, Faumont S, Coulthard SM, Thiele TR, Chronis N, McCormick KE, Goodman MB, Pruitt BL (2008) Artificial dirt: microfluidic substrates for nematode neurobiology and behavior. J Neurophysiol 99:3136-3143.

Marder E, Bucher D (2001) Central pattern generators and the control of rhythmic movements. Curr Biol 11:R986-R996.

Mattis J, Tye KM, Ferenczi EA, Ramakrishnan C, O'Shea DJ, Prakash R, Gunaydin LA, Hyun M, Fenno LE, Gradinaru V, Yizhar O, Deisseroth K (2011) Principles for applying optogenetic tools derived from direct comparative analysis of microbial opsins. Nat Methods 9:159-172.

Mclntire SL, Jorgensen E, Horvitz HR (1993a) Genes required for GABA function in Caenorhabditis elegans. Nature 364:334-337.

McIntire SL, Jorgensen E, Kaplan J, Horvitz HR (1993b) The GABAergic nervous system of Caenorhabditis elegans. Nature 364:337-341.

Mellem JE, Brockie PJ, Madsen DM, Maricq AV (2008) Action potentials contribute to neuronal signaling in C. elegans. Nat Neurosci 11:865-867.

Miller DM, 3rd, Niemeyer CJ (1995) Expression of the unc-4 homeoprotein in Caenorhabditis elegans motor neurons specifies presynaptic input. Development 121:2877-2886.

Miller DM, Shen MM, Shamu CE, Bürglin TR, Ruvkun G, Dubois ML, Ghee M, Wilson L (1992) C. elegans unc-4 gene encodes a homeodomain protein that determines the pattern of synaptic input to specific motor neurons. Nature 355:841-845.

Nagel G, Brauner M, Liewald JF, Adeishvili N, Bamberg E, Gottschalk A (2005) Light activation of channelrhodopsin-2 in excitable cells of 
Caenorhabditis elegans triggers rapid behavioral responses. Curr Biol 15:2279-2284.

Niebur E, Erdös P (1991) Theory of the locomotion of nematodes: Dynamics of undulatory progression on a surface. Biophys $\mathrm{J}$ 60:1132-1146.

Okazaki A, Sudo Y, Takagi S (2012) Optical silencing of C. elegans cells with arch proton pump. PLoS One 7:e35370.

Olivares EO, Izquierdo EJ, Beer RD (2018) Potential role of a ventral nerve cord central pattern generator in forward and backward locomotion in Caenorhabditis elegans. Netw Neurosci 2:323-343.

Petrash HA, Philbrook A, Haburcak M, Barbagallo B, Francis MM (2013) ACR-12 ionotropic acetylcholine receptor complexes regulate inhibitory motor neuron activity in Caenorhabditis elegans. J Neurosci 33:5524-5532.

Petzold BC, Park S-J, Ponce P, Roozeboom C, Powell C, Goodman MB, Pruitt BL (2011) Caenorhabditis elegans body mechanics are regulated by body wall muscle tone. Biophys J 100:1977-1985.

Pierce-Shimomura JT, Chen BL, Mun JJ, Ho R, Sarkis R, Mclntire SL (2008) Genetic analysis of crawling and swimming locomotory patterns in C. elegans. Proc Natl Acad Sci USA 105:20982-20987.

Pirri JK, McPherson AD, Donnelly JL, Francis MM, Alkema MJ (2009) A tyramine-gated chloride channel coordinates distinct motor programs of a Caenorhabditis elegans escape response. Neuron 62:526-538.

Ranner T (2020) A stable finite element method for low inertia undulatory locomotion in three dimensions. Appl Numer Math 156:422445.

Roberts A, Li WC, Soffe SR (2008) Roles for inhibition: studies on networks controlling swimming in young frog tadpoles. J Comp Physiol A Neuroethol Sens Neural Behav Physiol 194:185-193.

Satterlie RA (1985) Reciprocal inhibition and postinhibitory rebound produce reverberation in a locomotor pattern generator. Science 229:402-404.

Satou C, Kimura Y, Kohashi T, Horikawa K, Takeda H, Oda Y, Higashijima SI (2009) Functional role of a specialized class of spinal commissural inhibitory neurons during fast escapes in zebrafish. J Neurosci 29:6780-6793.

Schultheis C, Brauner M, Liewald JF, Gottschalk A (2011) Optogenetic analysis of GABAB receptor signaling in Caenorhabditis elegans motor neurons. J Neurophysiol 106:817-827.

Schuske K, Beg AA, Jorgensen EM (2004) The GABA nervous system in C. elegans. Trends Neurosci 27:407-414.

Sherrington CS (1906) Observations on the scratch-reflex in the spinal dog. J Physiol 34:1-50.

Sherrington CS (1908) Reciprocal innervation of antagonistic muscles. Thirteenth note.-On the antagonism between reflex inhibition and reflex excitation. Proc Royal Soc B 80:565-578.
Song J, Pallucchi I, Ausborn J, Ampatzis K, Bertuzzi M, Fontanel P, Picton LD, El Manira A (2020) Multiple rhythm-generating circuits act in tandem with pacemaker properties to control the start and speed of locomotion. Neuron 105:1048-1061.e4.

Stephens GJ, Johnson-Kerner B, Bialek W, Ryu WS (2008) Dimensionality and dynamics in the behavior of $C$. elegans. PLoS Comput Biol 4:e1000028.

Talpalar AE, Endo T, Lo w P, Borgius L, Hägglund M, Dougherty KJ, Ryge J, Hnasko TS, Kiehn O (2011) Identification of minimal neuronal networks involved in flexor-extensor alternation in the mammalian spinal cord. Neuron 71:1071-1084.

Tolstenkov O, Van der Auwera P, Costa WS, Bazhanova O, Gemeinhardt TM, Bergs AC, Gottschalk A (2018) Functionally asymmetric motor neurons contribute to coordinating locomotion of Caenorhabditis elegans. Elife 7:e34997.

Vidal-Gadea A, Topper S, Young L, Crisp A, Kressin L, Elbel E, Maples T, Brauner M, Erbguth K, Axelrod A, Gottschalk A, Siegel D, Pierce-Shimomura JT (2011) Caenorhabditis elegans selects distinct crawling and swimming gaits via dopamine and serotonin. Proc Natl Acad Sci USA 108:17504-17509.

Wen Q, Po MD, Hulme E, Chen S, Liu X, Kwok SW, Gershow M, Leifer AM, Butler V, Fang-Yen C, Kawano T, Schafer WR, Whitesides G, Wyart M, Chklovskii DB, Zhen M, Samuel AD (2012) Proprioceptive coupling within motor neurons drives $C$. elegans forward locomotion. Neuron 76:750-761.

White JG, Southgate E, Thomson JN, Brenner S (1976) The structure of the ventral nerve cord of Caenorhabditis elegans. Philos Trans $R$ Soc Lond B Biol Sci 275:327-348.

White JG, Southgate E, Thomson JN, Brenner S (1986) The structure of the nervous system of the nematode Caenorhabditis elegans. Philos Trans R Soc Lond B Biol Sci 314:1-340.

Wilson DM (1961) The central nervous control of flight in a locust. J Exp Biol 38:471-490.

Wu CY, Gagnon DA, Sardin JS, Barot U, Telenson A, Arratia PE, Kalb RG (2018) Enhancing GABAergic transmission improves locomotion in a Caenorhabditis elegans model of spinal muscular atrophy. eNeuro 5:ENEURO.0289-18.2018.

Xu T, Huo J, Shao S, Po M, Kawano T, Lu Y, Wu M, Zhen M, Wen Q (2018) Descending pathway facilitates undulatory wave propagation in Caenorhabditis elegans through gap junctions. Proc Natl Acad Sci USA 115:E4493-E4502.

Yanik MF, Cinar H, Cinar HN, Chisholm AD, Jin Y, Ben-Yakar A (2004) Neurosurgery: functional regeneration after laser axotomy. Nature 432:822.

Zhang J, Lanuza GM, Britz O, Wang Z, Siembab VC, Zhang Y, Velasquez T, Alvarez FJ, Frank E, Goulding M (2014) V1 and v2b interneurons secure the alternating flexor-extensor motor activity mice require for limbed locomotion. Neuron 82:138-150. 